\section{1}

\title{
Neural basis of opioid-induced respiratory depression and its rescue.
}

Shijia Liu ${ }^{1,2}$, Dongil Kim ${ }^{1}$, Tae Gyu Oh${ }^{3}$, Gerald $\mathrm{Pao}^{4}$, Jonghyun Kim ${ }^{1}$, Richard D. Palmiter ${ }^{5}$, Matthew R. Banghart ${ }^{2}$, Kuo-Fen Lee ${ }^{1,2}$, Ronald M. Evans ${ }^{3}$, Sung Han ${ }^{1,2, *}$

${ }^{1}$ Peptide Biology Laboratories, The Salk Institute for Biological Studies, La Jolla, CA 92037, USA.

${ }^{2}$ Section of Neurobiology, Division of Biological Sciences, University of California, San Diego, La Jolla, CA 92093, USA.

${ }^{3}$ Howard Hughes Medical Institute, Gene Expression Laboratories, The Salk Institute for Biological Studies, La Jolla, CA 92037, USA.

${ }^{4}$ Molecular and Cellular Biology Laboratories, The Salk Institute for Biological Studies, La Jolla, CA 92037, USA. ${ }^{5}$ Howard Hughes Medical Institute, Department of Biochemistry, School of Medicine, University of Washington, Seattle, WA 98195, USA.

*Corresponding author: Sung Han, $\mathrm{PhD}$

Author Contributions: S.H. conceived the idea, designed and constructed respiratory monitoring apparatus, and secured funding. S.H. and S.L. designed the experiments and wrote the manuscript with inputs from other authors. S.L. performed most of the experiments. D.K. and J.K. prepared the sample for RiboTag RNA sequencing. T.G.O. analyzed the data from RiboTag RNA sequencing. G.M.P. performed the CCM analysis. R.D.P. provided the Oprm1 ${ }^{\text {Cre }}$ mouse line. M.R.B. provided the hMOR virus. K.F.L. and R.M.E. provided experimental resources.

\section{Acknowledgments}

We thank the Han lab members for the critical discussion of the paper and D. O'Keefe and M. Shields for critical inputs on the manuscript. S.H. is supported by 1R01MH116203 from NIMH, the Bridge to Independence award from the Simons Foundation Autism Research Initiative (SFARI \#388708), and Brain Research Foundation Fay/Frank Seed Grant. K.F.L is supported by MH114831, OD023076, AG062232, NS115183 and AG064049.

Competing Interest Statement: The authors have declared no conflict of interest. 


\section{Abstract}

Opioid-induced respiratory depression (OIRD) causes death following an opioid overdose, yet the neurobiological mechanisms of this process are not well understood. Here, we show that neurons within the lateral parabrachial nucleus that express the $\mu$-opioid receptor ( $\mathrm{PBL}^{\text {Oprml }}$ neurons) are involved in OIRD pathogenesis. $\mathrm{PBL}^{\text {Oprml }}$ neuronal activity is tightly correlated with respiratory rate, and this correlation is abolished following morphine injection. Chemogenetic inactivation of $\mathrm{PBL}^{\text {Oprml }}$ neurons mimics OIRD in mice, whereas their chemogenetic activation following morphine injection rescues respiratory rhythms to baseline levels. We identified several excitatory G-protein coupled receptors expressed by $\mathrm{PBL}^{\text {Oprml }}$ neurons and show that agonists for these receptors restore breathing rates in mice experiencing OIRD. Thus, $\mathrm{PBL}^{\text {Oprml }}$ neurons are critical for OIRD pathogenesis, providing a promising therapeutic target for treating OIRD in patients.

\section{Introduction}

The misuse of prescription and recreational opioids has taken nearly 4.5 million lives between 1999 and 2018 in the United States alone (1). The direct cause of death from opioid overdose is opioid-induced respiratory depression (OIRD) $(2,3)$. Currently, the only available antidote capable of reversing OIRD is naloxone, a nonselective opioid receptor antagonist. However, naloxone is associated with several disadvantages, namely the reappearance of OIRD due to its short half-life, the inability to reverse high-affinity opioid drugs (e.g., carfentanil and buprenorphine) due to its low binding affinity, and the potential to induce a catecholamine surge at high doses, which can cause cardiopulmonary arrest (4-6). Alternative non-opioid interventions include respiratory stimulants that act on the brainstem respiratory network (e.g., ampakines) or oxygen-sensing cells within the carotid bodies (e.g., $\mathrm{K}^{+}$-channel blockers) (5). However, none of these alternatives are as effective as naloxone, and the safety of these interventions has not been well documented. Therefore, novel treatment strategies are needed, and more effective countermeasures can only be developed with a mechanistic understanding of OIRD pathogenesis.

Neural substrates that contribute to OIRD pathogenesis should satisfy two criteria. They should localize to the breathing control network and express the $\mu$-opioid receptor (MOR, encoded by the Oprml gene), which has been demonstrated as the primary mediator of both the analgesic and respiratory effects of opioids $(7,8)$. Two candidate structures satisfy these criteria, namely the pre-Bötzinger complex (preBötC) and the parabrachial complex (9-17). The former lies in the ventrolateral medulla and is the primary generator of the respiratory rhythm (18-20). The parabrachial complex, including the lateral parabrachial, medial parabrachial, and Kölliker-Fuse (KF) nuclei, is located in the dorsolateral pons and modulates breathing (21-23), in response to homeostatic disturbances 
such as $\mathrm{CO}_{2} / \mathrm{O}_{2}$ imbalance and noxious stimuli (24-28). Recent studies have suggested the involvement of these two brain regions on OIRD pathogenesis by using either pharmacological or genetic approaches. Direct infusion of opioid agonists or antagonists into these brain areas induces or attenuates $\operatorname{OIRD}(9,12,29$, 30). Region-specific genetic deletion of the Oprm1 genes by viral delivery of Cre-recombinase into these areas of the Oprm1-floxed mice also attenuates $\operatorname{OIRD}(13,14)$. However, the pharmacological approach lacks considerable specificity primarily due to the local diffusion of drugs and the difficulty of reproducible targeting, whereas the genetic deletion approach is often incomplete and prevents the possibility to directly control the neuronal activity in a spatially and temporally precise manner. As a result, discrepancies arise over the critical site of action responsible for OIRD (13, $14,16,17)$. To expand the scope of current research, our paper utilizes contemporary cell-type-specific approaches to characterize the role of a defined neuronal population in morphine-induced respiratory depression. This will allow us to better understand its cellular and physiological mechanisms and make inroads in developing therapeutic strategies.

Here we report that a genetically defined population of neurons that encodes the Oprml gene in the lateral 71 parabrachial nucleus ( $\mathrm{PBL}^{\text {Oprml }}$ neurons) is an important regulator of respiratory rhythm. Inhibition of these neurons 72 by opioids leads to respiratory depression. Furthermore, we show that both chemogenetic activation of these neurons 73 and pharmacological activation of endogenous G-protein coupled receptors (GPCRs) expressed specifically in these neurons completely rescues OIRD in mice. 


\section{Results}

To examine the contributions of global MOR signaling to OIRD, we generated an Oprm1 reporter mouse line in which the endogenous Oprml gene was knocked out by inserting a Cre:GFP cassette upstream of the start codon $\left(\mathrm{OprmI}^{\mathrm{Cre}}\right)$ (Figure 1B and Supplementary Figure 1A for validation), and measured the effects of systemic morphine injection on breathing rhythms via whole-body plethysmography (Figure 1A). After receiving a morphine injection, wild-type mice exhibited a significantly lower respiratory rate $(46.5 \pm 3.1 \%$ of the pre-morphine baseline (data are represented as mean \pm SEM throughout the paper)). In contrast, mice lacking one or both functional copies of the $\mathrm{Oprml}$ gene $\left(\mathrm{Oprml}^{\mathrm{Cr} /+}\right.$ and $\left.\mathrm{Oprml}^{\mathrm{Cr} / \mathrm{Cre}}\right)$ exhibited more moderate reductions in respiratory rate $(63.2 \pm$ $2.8 \%$ and $91.2 \pm 2.8 \%$ of the pre-morphine baseline, respectively) (Figure 1C, D). Oprm1, therefore, plays a vital role in OIRD.

We then used pharmacological and genetic tools to manipulate opioid signaling specifically in the PBL. First, wild-type mice received a systemic injection of morphine followed by an infusion of naloxone directly into the PBL (Figure 1E). To facilitate stereotaxic naloxone delivery, we lightly anesthetized mice with 1-1.5\% isoflurane and monitored their breathing using a piezoelectric sensor that detects chest movements (31). After systemic injection of morphine, the breathing rate dramatically decreased from $121.0 \pm 3.4 \mathrm{bpm}$ to $89.1 \pm 3.5 \mathrm{bpm}$, and subsequent stereotaxic injection of naloxone into the PBL recovered the breathing rate back to pre-morphine baseline $(120.8 \pm 3.7 \mathrm{bpm}$ ) (Figure 1F, G). We then explored whether the expression of the Oprm1 gene in the PBL is necessary and sufficient for OIRD. To assess necessity, we conditionally knocked out the Oprml gene using stereotaxic delivery of a recombinant adeno-associated virus (AAV) expressing Cre recombinase into the PBL of the $O p r m 1^{f l f l}$ mice, in which the Oprml gene is flanked by loxP sites (Figure 1H). Compared to mice receiving GFP-expressing control virus, PBL-specific knockout of the Oprm1 gene attenuated OIRD following systemic injection of morphine, as measured by whole-body plethysmography (breathing rate changes in GFP group: -136.6 $\pm 21.1 \mathrm{bpm}$; Cre group: $-76.4 \pm 11.6 \mathrm{bpm}$ ) (Figure 1I and Supplementary Figure 2A-C). To test sufficiency, we reintroduced the wild-type Oprml gene into the $\mathrm{PBL}^{\text {Oprml }}$ neurons of Cre-expressing Oprml-null mice. This was achieved by injecting an AAV expressing Cre-dependent human MOR (AAV-hSyn-DIO-mCherry-T2A-FLAGhMOR) (Figure 1J) into the PBL of Oprm1 $1^{\text {Cre/Cre }}$ mice. Selective expression of hMOR in the PBL restored the OIRD phenotype (hMOR group, post-saline: $359.0 \pm 5.1 \mathrm{bpm}$; post-morphine: $299.0 \pm 3.6 \mathrm{bpm}$ ) compared to the control group (Figure 1K and Supplementary Figure 2D). Note that the breathing rate measured by the piezoelectric sensor under anesthesia (Figure $1 \mathrm{G}$ ) is $\sim 3$-fold lower than that measured in a plethysmography chamber (Figure $1 \mathrm{~K})$. Together, these data strongly support the conclusion that the Oprml gene expression in the PBL mediates OIRD in mice. 
We then focused on PBL ${ }^{\text {Oprml }}$ neurons and explored their relationship with OIRD. PBL ${ }^{\text {Oprml }}$ neurons are mostly glutamatergic and express higher levels of the Oprml gene than the adjacent KF nucleus, which provides post-inspiratory drive $(13,23,31,32)$ (Supplementary Figure 1B-C). We devised an experimental platform to simultaneously measure $\mathrm{PBL}^{\text {Oprm }}$ neuronal activity and respiration in awake, freely moving mice using fiberphotometry calcium imaging in vivo with a thermistor implanted into the nasal cavity (Figure 2A). The thermistorbased respiration monitoring device detects the temperature change between inhaled and exhaled air and converts breath-to-breath fluctuations in resistance into voltage signals (33). We stereotaxically injected an AAV expressing 114 Cre-dependent jGCaMP7s (AAV-DIO-jGCaMP7s) into the PBL, then implanted an optic fiber into the PBL of $115 \mathrm{Oprml}^{\mathrm{Cre} / \mathrm{+}}$ mice and a respiration sensor in the nasal cavity (Figure 2B). $\mathrm{PBL}^{\text {Oprml }}$ activity and breathing rate 116 exhibited a tight positive correlation (Figure 2C-D, H). Moreover, morphine administration substantially decreased 117 the breathing rate $(46.5 \pm 3.9 \%$ of the pre-morphine baseline) (Figure $2 \mathrm{H}, \mathrm{J}, \mathrm{L}$, and $\mathrm{N}$ ) despite the potent effect 118 morphine has on locomotor sensitization (Supplementary Figure 3), which one would expect to elevate the breathing rate. Besides, morphine decreased calcium activity (Figure 2H, J, O, and Q), as well as eliminated any spontaneous fluctuations in both breathing and calcium signals (Figure 2N, Q). In contrast, saline injection largely preserved the original patterns of the signals (Figure 2D, 2F, 2L-M, and 2O-P). Using convergent cross-mapping (CCM) (34), a statistical algorithm that makes predictions from independent time-series data, we predicted changes in respiratory rate using the calcium signal as input $(76.2 \pm 8.1 \%$, pre-morphine predictability) (Supplementary Figure 4). Interestingly, systemic morphine administration not only reduced the correlation coefficient (Figure 2I, K) but also completely abolished the predictive relationship between respiratory rate and the calcium signal $(12.5 \pm 20.2 \%$, post-morphine predictability) (Supplementary Figure 4C-D). Conversely, the saline injection did not alter the correlation between these signals (Fig 2E, G, and Supplementary Figure 4A-B). These results indicate that PBL ${ }^{\text {Oprml }}$ neurons are critically involved in modulating respiratory rate and that morphine suppresses both the $\mathrm{PBL}^{\text {Oprml }}$ neuronal activity and its coupling with the breathing rate.

To further investigate $\mathrm{PBL}^{\text {Oprml }}$ neurons' role in breathing regulation, we specifically manipulated their activity using chemogenetic tools, namely Designer Receptor Exclusively Activated by Designer Drugs

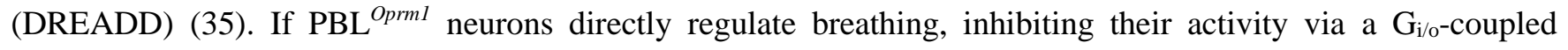
DREADD should recapitulate the OIRD phenotype. Furthermore, this should also happen in Oprml knockout mice, which failed to display OIRD when given systemic morphine (Figure 1C-D). We expressed a א-opioid receptorderived DREADD (KORD) (36) Cre-dependently and bilaterally in $\mathrm{PBL}^{\text {Oprml }}$ neurons of $\mathrm{Oprm} 1^{\mathrm{Cre} / \mathrm{Cre}}$ mice (Figure 3A). Systemic injection of its synthetic ligand, salvinorin B (SALB), decreased the respiratory rate $(317.8 \pm 3.6$ $137 \mathrm{bpm}$ ) compared to the DMSO-injected control group (342.4 $\pm 4.1 \mathrm{bpm}$ ) (Figure 3B and Supplementary Figure 5A138 B). We did not observe significant breathing amplitude changes upon SALB injection in either control or KORDexpressing animals (Supplementary Figure 5B-C). 
We next asked whether artificial activation of $\mathrm{PBL}^{\text {Oprml }}$ neurons could prevent OIRD in mice. We expressed hM3Dq, a metabotropic acetylcholine receptor-derived excitatory DREADD, in $\mathrm{PBL}^{\text {oprml }}$ neurons via bilateral injection of an AAV encoding Cre-dependent hM3Dq into Oprm $1^{\mathrm{Cre} /+}$ mice (Figure 3C). Additionally, we implanted a micro-thermistor sensor into the nasal cavity to monitor respiratory rhythm in awake, freely moving mice over the course of OIRD (Supplementary Figure 5D). Upon systemic morphine injection, the respiratory rate decreased significantly within $10 \mathrm{~min}$ (from $332.0 \pm 25.6 \mathrm{bpm}$ to $124.6 \pm 9.1 \mathrm{bpm}$ ) (Figure 3D-E). We then systemically injected the hM3Dq agonist, clozapine-N-oxide (CNO), into the same animal to activate $\mathrm{PBL}^{\text {Oprml }}$ neurons. CNO injection increased the breathing rate in $\mathrm{hM} 3 \mathrm{Dq}$-expressing animals $(268.0 \pm 13.5 \mathrm{bpm})$ to a level comparable to controls that did not receive morphine $(275.7 \pm 24.9 \mathrm{bpm})$. Nevertheless, CNO did not rescue breathing rates in eYFP-expressing control animals $(120.0 \pm 4.3 \mathrm{bpm})$ (Figure 3D-F and Supplementary Figure 5E). A complete rescue was also observed in minute volume (Supplementary Figure 5F, G). These data show that activation of $\mathrm{PBL}^{\text {Oprml }}$ neurons is sufficient to restore normal breathing rhythms in mice displaying OIRD.

Having determined that chemogenetic activation of $\mathrm{PBL}^{\text {Oprml }}$ neurons could effectively prevent OIRD, we sought to identify endogenous signaling pathways that could be used to activate $\mathrm{PBL}^{\text {oprml }}$ neurons. To identify the active transcriptome enriched in $\mathrm{PBL}^{\text {Oprml }}$ neurons, we sequenced ribosome-associated mRNAs from the PBL of Oprm1 $^{\mathrm{Cre}}:$ :RiboTag mice, which express hemagglutinin (HA)-tagged ribosomal protein Rpl22 in Oprm $^{+}$neurons (Figure 4A-B) (37). We identified 69 mRNAs upregulated over three folds in PBL ${ }^{\text {Oprm1 }}$ neurons than non-Oprm1 neurons in the PBL (Figure 4C). Oprm1, as well as other major parabrachial markers (Tac1, Foxp2, and Adcyap1) were enriched, but Calca and Pdyn, markers for the external lateral and dorsal lateral parabrachial nucleus, were not $(38,39)$. Notably, the $\mathrm{PBL}^{\text {oprml }}$ active transcriptome revealed several GPCRs expressed at high levels in these neurons, which we subsequently investigated as potential pharmacological targets to rescue OIRD.

We selected five excitatory GPCRs for pharmacological manipulation and confirmed that they were expressed in PBL ${ }^{\text {Oprm }}$ neurons by RNA in situ hybridization (Supplementary Figure 6A-B). These GPCRs were 5hydroxytryptamine receptor 2A (Htr2a), cholecystokinin A receptor (Cckar), tachykinin receptor 1 (Tacrl), tachykinin receptor 3 (Tacr3), and dopamine receptor D5 (Drd5). We systemically injected their agonists (TCB-2, CCK8S, Substance P, Senktide, and SKF83959, respectively) into the PBL of anesthetized mice after inducing OIRD (breathing rate before morphine: $115.4 \pm 1.6 \mathrm{bpm}$, after morphine: $77.9 \pm 2.3 \mathrm{bpm}$; Figure 4D-E and

167 Supplementary Table 1). Three of the five agonists tested (TCB-2, CCK8S, and Substance P) increased the 168 respiratory rate $(98.1 \pm 7.9,117.1 \pm 6.4,100.8 \pm 4.6 \mathrm{bpm}$; Figure 4F and Supplementary Figure 6C-E). Notably, these same drugs have been shown to modulate respiratory rhythm in different contexts by activating other respiratory centers, and some have been associated with an anti-morphine effect (40-42). Our findings suggest that activating $\mathrm{PBL}^{\text {Oprml }}$ neurons through endogenous signaling pathways may effectively treat OIRD in patients. 


\section{Discussion}

We have identified a population of neurons in the pontine respiratory group, the $\mathrm{PBL}^{\text {oprml }}$ neurons, as critical mediators of morphine-induced respiratory depression and candidates for its rescue in intact mice. Genetic deletion of Oprm1 from the PBL attenuated OIRD, while reintroducing human MOR into the PBL ${ }^{\text {Oprml }}$ neurons restored OIRD. By combining cell-type-specific tools with breathing monitoring in awake behaving mice, we have demonstrated that $\mathrm{PBL}^{\text {oprm }}$ neurons are regulators of the breathing rhythm and pattern. Systemic morphine administration dramatically abolishes the activity of these neurons and weakens their control on breathing. Furthermore, chemogenetic inhibition of $\mathrm{PBL}^{\text {Oprml }}$ neurons mimics OIRD, and prolonged optogenetic inhibition of $\mathrm{PBL}^{\text {oprml }}$ neurons induces apnea (observations from our group (43)), which precedes cardiorespiratory collapse prior to overdose-induced death. By contrast, artificial activation of $\mathrm{PBL}^{\text {Oprml }}$ neurons, either chemogenetically or pharmacologically, successfully restores breathing in mice experiencing OIRD (Supplementary Figure 7).

\section{Investigating OIRD with cell-type-specific approaches.}

Recent studies have demonstrated the involvement of both pontine and medullary respiratory groups in OIRD $(13,14)$ without reaching a consensus on the key players. We think this is mostly due to the lack of specificity from the pharmacological and genetic deletion approaches and the intricacies of physiological preparations, both of which hampers reproducibility. In comparison, we exploited the $\mathrm{Oprm} 1^{\mathrm{Cre}}$ mouse line which provides access to a restricted neuronal population, and obtained multi-faceted evidence supporting the role of the parabrachial nucleus in OIRD. In our view, OIRD is a synergistic outcome from impairment of the interconnected pontomedullary breathing network that widely expresses MOR both pre- and post-synaptically $(10,11,30,44,45)$. For example, parabrachial neurons send tonic excitatory inputs to the preBötC $(39,43,46,47)$, and both of these areas express $\operatorname{MOR}(18,48)$.

One challenge of elucidating the OIRD mechanism is how to access a group of neurons while simultaneously record breathing over the course of OIRD. Traditional respiratory monitoring approaches in rodents, such as in situ or in vivo anesthetized preparations and plethysmography chambers, are more challenging to achieve this goal. Therefore, we utilized the implantable temperature sensor to monitor breathing in awake, unrestrained, behaving mice with unprecedented freedom to access the brain due to its open configuration. With this approach, we implemented chemogenetics and in vivo calcium imaging while simultaneously monitored breathing rhythms. Similar techniques can be applied to the Oprml-expressing neurons in the preBötC, KF, the postinspiratory complex (49), and other respiratory centers to resolve the long-standing debate on their contributions in OIRD $(16,17)$.

\section{PBL $^{\text {Oprml }}$ neurons' role in breathing regulation and OIRD.}


The parabrachial complex has long been reported to modulate respiration and sometimes exert opposing effects depending on the location of artificial manipulation (21-23). Stimulation of the PBL results in tachypnea, whereas stimulation of the KF elicits prolonged post-inspiration and bradypnea $(13,31,32)$. Consistent with these previous reports, our monitoring and manipulation data suggest that $\mathrm{PBL}^{\text {oprml }}$ neurons have the most substantial effect on the breathing rate (23). In addition to breathing, the PBL also regulates various homeostatic functions (39) and incorporates the alarm center, which senses deviations from the homeostasis and relays aversive signals to higher-order brain structures $(38,50)$. At the same time, by sending tonic excitatory outputs to medulla respiratory centers $(39,43,46)$, the parabrachial neurons can increase breathing rhythm under conditions that immediately require more oxygen due to metabolic needs, such as hypoxia (27) and hypercapnia (26), in particular, hypercapnic arousal during sleep $(24,28,51)$; or non-metabolic behavioral demands, such as escaping from a threat (25). As a representative glutamatergic population in the PBL (Supplementary Figure 1), Oprm1-positive neurons are likely to participate in similar physiological processes. Our data demonstrated that an overdose of morphine shuts down $\mathrm{PBL}^{\text {Oprml }}$ neurons' activity, preventing them from responding to conditions that decrease respiratory rhythm. These conditions may include hypoxia, hypercapnia, anesthesia, or sedative drug treatment. Indeed, the incidence of OIRD is dramatically increased when opioids are used together with sedatives and anesthetics (52), and the failure to increase respiratory behavior in response to hypercapnic gas is one characteristic of OIRD (3). On the contrary, a mild inhibition of $\mathrm{PBL}^{\text {oprm }}$ neurons may explain many of the behavioral effects of lower doses of endogenous or exogenous opioids, such as slowed breathing, calmness, and reward (43). The current study used morphine as a starting point to induce OIRD, and further investigation is required to determine the involvement of $\mathrm{PBL}^{\text {Oprml }}$ neurons under the challenge of other types of sedative agents.

\section{A proof of principle of rescuing OIRD by activating a pontine population.}

$\mathrm{PBL}^{\text {Oprml }}$ neurons are among the key nodes most vulnerable to opioid action, and their activation is sufficient to restore the breathing rate and tidal volume following morphine administration. This activation can be achieved by exploiting both artificial and, more importantly, endogenous signaling pathways. Besides, manipulating $\mathrm{PBL}^{\text {Oprml }}$ neurons located in the dorsolateral pons is associated with lower risk than the respiratory 227 rhythm generators within the deep medulla. We have provided a proof of concept of rescuing OIRD by activating 228 endogenous $\mathrm{G}_{\mathrm{q} / \mathrm{s}}$-coupled GPCRs expressed in PBL ${ }^{\text {Oprml }}$ neurons (Supplementary Figure 7). In principle, cell-type229 specific manipulation can also be achieved through the collective activation of multiple GPCRs. 
233 hypercapnia-induced arousal by integrating serotonergic inputs from the dorsal raphe (51). It would be interesting 234 to identify other neuromodulatory circuits that may be involved in the regulation of OIRD. Similar transcriptomic 235 profiling strategies can also be applied to the broader breathing control network to exploit the therapeutic potential 236 of GPCRs in reversing OIRD. 


\section{References}

267 1. H. Hedegaard, A. M. Miniño, M. Warner, Drug overdose deaths in the United States, 1999-2018. NCHS data brief 356 (2020).

$2692 . \quad$ K. T. Pattinson, Opioids and the control of respiration. Br. J. Anaesth. 100, 747-758 (2008).

$2703 . \quad$ A. Dahan, L. Aarts, T. W. Smith, Incidence, reversal, and prevention of opioid-induced respiratory 271 depression. Anesthesiology 112, 226-238 (2010).

272 4. P. Skolnick, On the front lines of the opioid epidemic: rescue by naloxone. European journal of 273 pharmacology 835, 147-153 (2018).

274 5. A. Dahan et al., Averting opioid-induced respiratory depression without affecting analgesia. Anesthesiology 275 128, 1027-1037 (2018).

276 6. E. W. Boyer, Management of opioid analgesic overdose. N. Engl. J. Med. 367, 146-155 (2012).

277 7. H. W. Matthes et al., Loss of morphine-induced analgesia, reward effect and withdrawal symptoms in mice lacking the $\mu$-opioid-receptor gene. Nature 383, 819 (1996).

8. A. Dahan et al., Anesthetic Potency and Influence of Morphine and Sevoflurane on Respiration in $\mu$-Opioid Receptor Knockout Mice. Anesthesiology 94, 824-832 (2001).

9. J. R. Miller et al., A Subregion of the Parabrachial Nucleus Partially Mediates Respiratory Rate Depression from Intravenous Remifentanil in Young and Adult Rabbits. Anesthesiology 127, 502-514 (2017).

10. E. Cinelli, F. Bongianni, T. Pantaleo, D. Mutolo, Activation of $\mu$-opioid receptors differentially affects the preBötzinger Complex and neighbouring regions of the respiratory network in the adult rabbit. Respir. Physiol. Neurobiol. 280, 103482 (2020).

11. A. D. Wei, J.-M. Ramirez, Presynaptic mechanisms and KCNQ potassium channels modulate opioid depression of respiratory drive. Front. Physiol. 10, 1407 (2019).

12. I. Prkic et al., Pontine mu-opioid receptors mediate bradypnea caused by intravenous remifentanil infusions at clinically relevant concentrations in dogs. J. Neurophysiol. 108, 2430-2441 (2012).

13. A. G. Varga, B. T. Reid, B. L. Kieffer, E. S. Levitt, Differential impact of two critical respiratory centres in opioid-induced respiratory depression in awake mice. The Journal of Physiology 598, 189-205 (2020).

14. I. Bachmutsky, X. P. Wei, E. Kish, K. Yackle, Opioids depress breathing through two small brainstem sites. Elife 9, e52694 (2020).

15. G. Montandon et al., PreBotzinger complex neurokinin-1 receptor-expressing neurons mediate opioidinduced respiratory depression. J. Neurosci. 31, 1292-1301 (2011).

16. G. Montandon, R. Horner, CrossTalk proposal: The preBotzinger complex is essential for the respiratory depression following systemic administration of opioid analgesics. J. Physiol. 592, 1159-1162 (2014). 
17. P. M. Lalley, P. M. Pilowsky, H. V. Forster, E. J. Zuperku, CrossTalk opposing view: The pre-Botzinger complex is not essential for respiratory depression following systemic administration of opioid analgesics. J. Physiol. 592, 1163-1166 (2014).

18. C. A. Del Negro, G. D. Funk, J. L. Feldman, Breathing matters. Nat. Rev. Neurosci. 19, 351-367 (2018).

19. J. C. Smith, H. H. Ellenberger, K. Ballanyi, D. W. Richter, J. L. Feldman, Pre-Bötzinger complex: a brainstem region that may generate respiratory rhythm in mammals. Science 254, 726-729 (1991).

20. Y. Cui et al., Defining preBotzinger Complex Rhythm- and Pattern-Generating Neural Microcircuits In Vivo. Neuron 91, 602-614 (2016).

21. N. L. Chamberlin, C. B. Saper, Topographic organization of respiratory responses to glutamate microstimulation of the parabrachial nucleus in the rat. J. Neurosci. 14, 6500-6510 (1994).

22. M. Dutschmann, T. E. Dick, Pontine mechanisms of respiratory control. Compr Physiol 2, 2443-2469 (2012).

23. A. A. Navarrete-Opazo et al., Endogenous glutamatergic inputs to the Parabrachial Nucleus/Kölliker-Fuse Complex determine respiratory rate. Respir. Physiol. Neurobiol. 277, 103401 (2020).

24. S. Kaur et al., A Genetically Defined Circuit for Arousal from Sleep during Hypercapnia. Neuron 96, 11531167 e1155 (2017).

25. M. Jiang, G. F. Alheid, T. Calandriello, D. R. McCrimmon, Parabrachial-lateral pontine neurons link nociception and breathing. Respir. Physiol. Neurobiol. 143, 215-233 (2004).

26. G. Song, C. S. Poon, Lateral parabrachial nucleus mediates shortening of expiration and increase of inspiratory drive during hypercapnia. Respir. Physiol. Neurobiol. 165, 9-12 (2009).

27. G. Song, C. S. Poon, Lateral parabrachial nucleus mediates shortening of expiration during hypoxia. Respir. Physiol. Neurobiol. 165, 1-8 (2009).

28. S. Kaur et al., Glutamatergic signaling from the parabrachial nucleus plays a critical role in hypercapnic arousal. J. Neurosci. 33, 7627-7640 (2013).

29. S. E. Saunders, E. S. Levitt, Kölliker-Fuse/Parabrachial complex mu opioid receptors contribute to fentanylinduced apnea and respiratory rate depression. Respir. Physiol. Neurobiol. 275, 103388 (2020).

30. P. M. Lalley, Opiate slowing of feline respiratory rhythm and effects on putative medullary phaseregulating neurons. Am. J. Physiol. Regul. Integr. Comp. Physiol. 290, R1387-1396 (2006).

31. E. S. Levitt, A. P. Abdala, J. F. Paton, J. M. Bissonnette, J. T. Williams, mu opioid receptor activation hyperpolarizes respiratory-controlling Kolliker-Fuse neurons and suppresses post-inspiratory drive. $J$. Physiol. 593, 4453-4469 (2015). 
32. M. Dutschmann, H. Herbert, The Kolliker-Fuse nucleus gates the postinspiratory phase of the respiratory cycle to control inspiratory off-switch and upper airway resistance in rat. Eur. J. Neurosci. 24, 1071-1084 (2006).

33. S. S. McAfee et al., Minimally invasive highly precise monitoring of respiratory rhythm in the mouse using an epithelial temperature probe. J. Neurosci. Methods 263, 89-94 (2016).

34. G. Sugihara et al., Detecting Causality in Complex Ecosystems. Science 338, 496-500 (2012).

35. B. L. Roth, DREADDs for Neuroscientists. Neuron 89, 683-694 (2016).

36. E. Vardy et al., A new DREADD facilitates the multiplexed chemogenetic interrogation of behavior. Neuron 86, 936-946 (2015).

37. E. Sanz, J. C. Bean, D. P. Carey, A. Quintana, G. S. McKnight, RiboTag: Ribosomal Tagging Strategy to Analyze Cell-Type-Specific mRNA Expression In Vivo. Curr. Protoc. Neurosci. 88, e77 (2019).

38. R. D. Palmiter, The Parabrachial Nucleus: CGRP Neurons Function as a General Alarm. Trends Neurosci. 41, 280-293 (2018).

39. D. Huang, F. S. Grady, L. Peltekian, J. C. Geerling, Efferent Projections of Vglut2, Foxp2 and Pdyn Parabrachial Neurons in Mice. J. Comp. Neurol. https://doi.org/10.1002/cne.24975 (2020).

40. M. Niebert et al., Expression and function of serotonin 2A and 2B receptors in the mammalian respiratory network. PLoS One 6, e21395 (2011).

41. H. H. Ellenberger, F. M. Smith, Sulfated cholecystokinin octapeptide in the rat: pontomedullary distribution and modulation of the respiratory pattern. Can. J. Physiol. Pharmacol. 77, 490-504 (1999).

42. J. Hedner, T. Hedner, P. Wessberg, J. Jonason, Interaction of substance P with the respiratory control system in the rat. Journal of Pharmacology and Experimental Therapeutics 228, 196-201 (1984).

43. S. Liu et al., Identification of a novel breathing circuit that controls pain and anxiety. bioRxiv https://doi.org/10.1101/2020.01.09.900738 (2020).

44. U. Arvidsson et al., Distribution and targeting of a mu-opioid receptor (MOR1) in brain and spinal cord. $J$. Neurosci. 15, 3328-3341 (1995).

45. M. A. Hurlé, A. Mediavilla, J. Florez, Differential respiratory patterns induced by opioids applied to the ventral medullary and dorsal pontine surfaces of cats. Neuropharmacology 24, 597-606 (1985).

46. S. Yokota, S. Kaur, V. G. VanderHorst, C. B. Saper, N. L. Chamberlin, Respiratory-related outputs of glutamatergic, hypercapnia-responsive parabrachial neurons in mice. J. Comp. Neurol. 523, 907-920 (2015).

47. C. E. Fulwiler, C. B. Saper, Subnuclear organization of the efferent connections of the parabrachial nucleus in the rat. Brain Res. Rev. 7, 229-259 (1984). 
361 48. E. Erbs et al., A mu-delta opioid receptor brain atlas reveals neuronal co-occurrence in subcortical networks. Brain Struct. Funct. 220, 677-702 (2015).

363 49. T. M. Anderson et al., A novel excitatory network for the control of breathing. Nature 536, 76-80 (2016).

$36450 . \quad$ K. Tokita, T. Inoue, J. D. Boughter, Afferent connections of the parabrachial nucleus in C57BL/6J mice. $365 \quad$ Neuroscience 161, 475-488 (2009).

366 51. S. Kaur et al., Role of serotonergic dorsal raphe neurons in hypercapnia-induced arousals. Nature 367 Communications 11, 1-15 (2020).

368 52. K. Gupta et al., Risk factors for opioid-induced respiratory depression and failure to rescue: a review. Curr. Opin. Anaesthesiol. 31, 110-119 (2018).

370 53. G. Sugihara, R. M. May, Nonlinear forecasting as a way of distinguishing chaos from measurement error 371 in time series. Nature 344, 734-741 (1990).

372 54. E. R. Deyle, G. Sugihara, Generalized theorems for nonlinear state space reconstruction. PLoS One 6, 373 e18295(2011).

$37455 . \quad$ H. Whitney, The imbedding of manifolds in families of analytic manifolds. Annals of Mathematics, 865$375878(1936)$.

376 56. A. M. Fraser, H. L. Swinney, Independent coordinates for strange attractors from mutual information. Phys 377 Rev A Gen Phys 33, 1134-1140 (1986).

378 57. E. Sanz et al., Cell-type-specific isolation of ribosome-associated mRNA from complex tissues. 379 Proceedings of the National Academy of Sciences 106, 13939-13944 (2009).

58. A. Dobin et al., STAR: ultrafast universal RNA-seq aligner. Bioinformatics 29, 15-21 (2013).

59. B. Li, C. N. Dewey, RSEM: accurate transcript quantification from RNA-Seq data with or without a reference genome. BMC Bioinformatics 12, 323 (2011). 
A

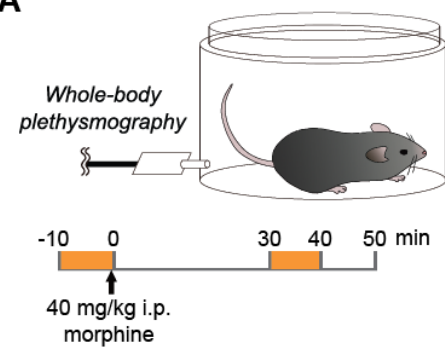

E

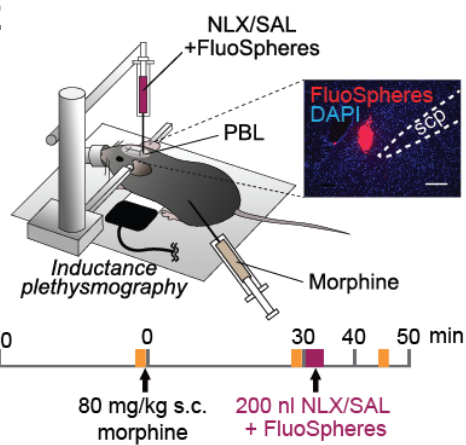

NLXISAL

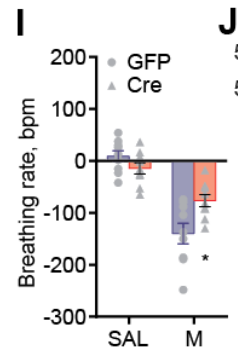

H

5' CAG

5' CAG $\mid$ GFP $\mid$ WPRE $\mid$ PA $3^{\prime}$
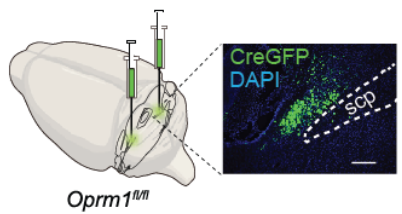

$\mathbf{F}$
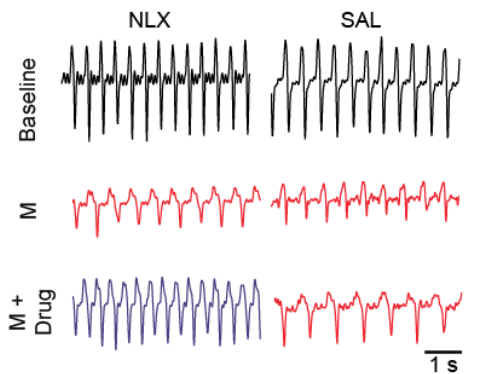

B

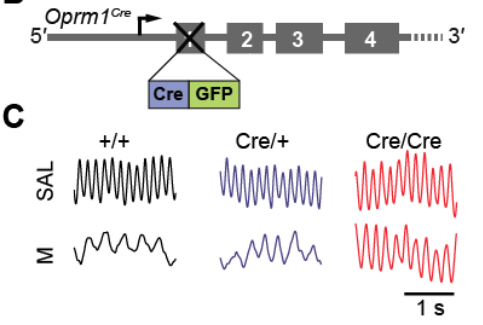

G

G
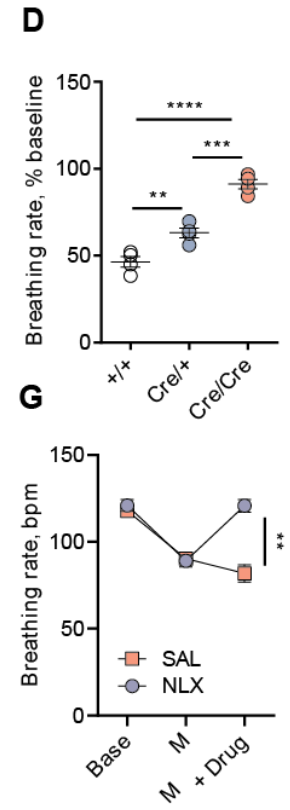

K

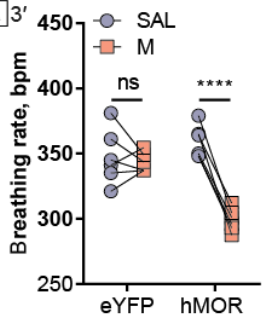

J

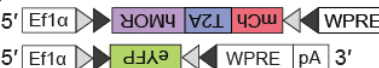

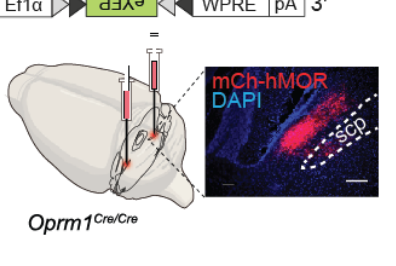

Figure 1. MOR signaling in the PBL mediates morphine-induced respiratory depression. (A) Schematics of breathing monitoring in awake mice with whole-body plethysmography before and after systemic morphine

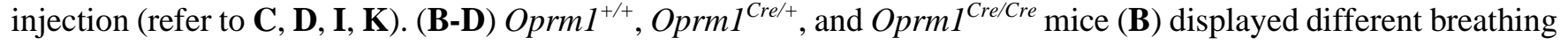
patterns $(\mathbf{C})$ and rate changes (D) in response to systemic morphine injection. (E) Schematics of breathing monitoring in anesthetized mice with inductance plethysmography, during systemic morphine injection and PBLspecific blockade of MOR signaling. Onset, histology of the co-injected FluoSpheres in the PBL. Scp, superior cerebellar peduncle. (F) Representative plethysmographs from naloxone (NLX) and saline (SAL) injected groups before morphine, after morphine, and after drug injections into the PBL. (G) Injecting naloxone ( $=5$ ), but not saline $(n=5)$ into the PBL rescued morphine-induced respiratory depression. (H) Schematics of PBL-specific knockout of the Oprml gene by stereotaxically injecting AAV-Cre-GFP into the PBL of the Oprm $1^{f l f l}$ mice. (I) PBL-specific Oprml knockout $(\mathrm{n}=9)$ significantly attenuated OIRD compared to the GFP injected control group $(\mathrm{n}=9)$ after systemic morphine injection. (J) Schematics of PBL-specific rescue of the Oprm1 gene in Oprm1-null

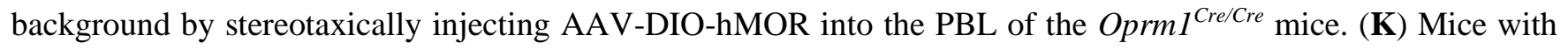
PBL-specific rescue of Oprml $(\mathrm{n}=6)$ displayed OIRD phenotype, whereas eYFP injected control group $(\mathrm{n}=6)$ remained insensitive after systemic morphine injection. Data are presented as mean \pm SEM. Statistical tests, Oneway ANOVA with Tukey's multiple comparison post-hoc test (D) or Two-way ANOVA with Bonferroni's multiple comparison post-hoc test $(\mathbf{G}, \mathbf{I}, \mathbf{K}) .^{*}, p<0.05 ; * *, p<0.01$; ***, $p<0.001$; ****, $p<0.0001$. Scale bar, $1 \mathrm{~s}(\mathbf{C}, \mathbf{F})$ or $200 \mu \mathrm{m}(\mathbf{E}, \mathbf{H}, \mathbf{J})$. 
A
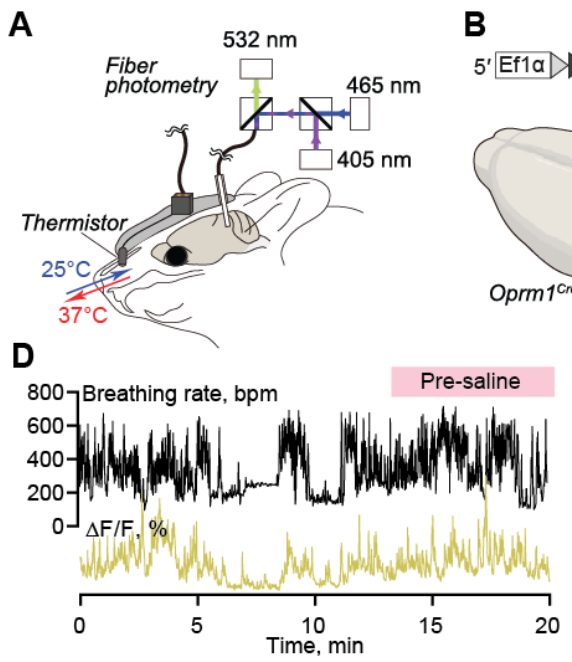

H
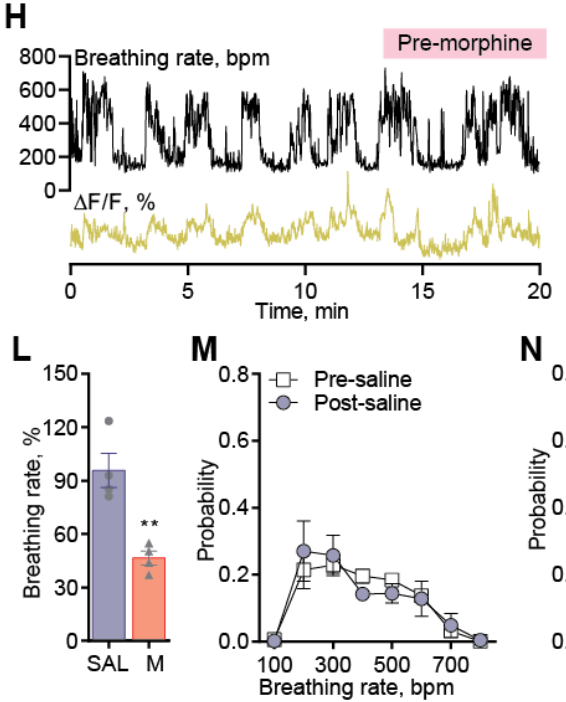

B

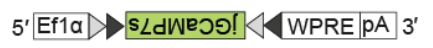

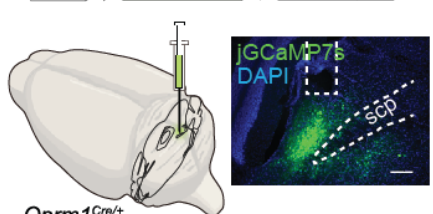

E

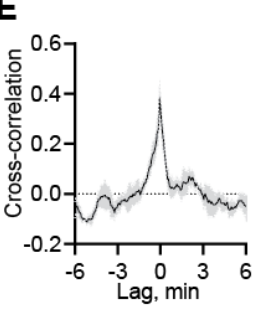

I

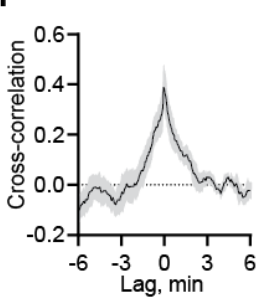

$\mathbf{N}_{0}$

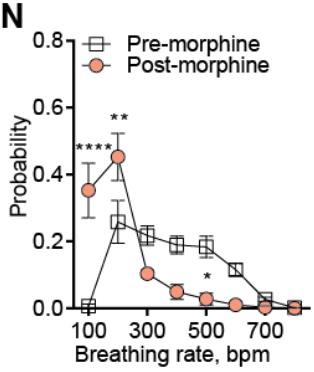

J
C

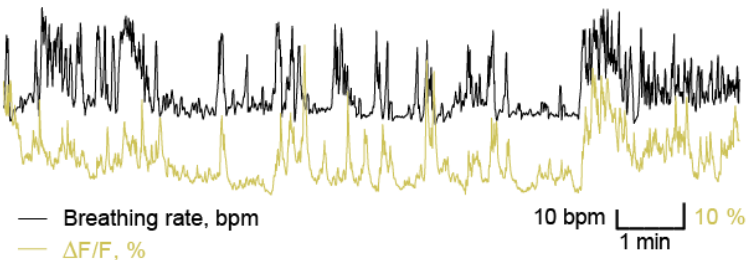

F

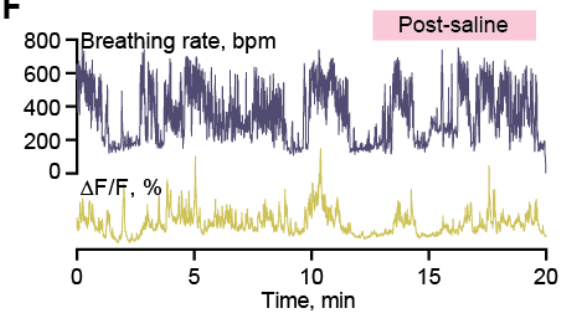

G
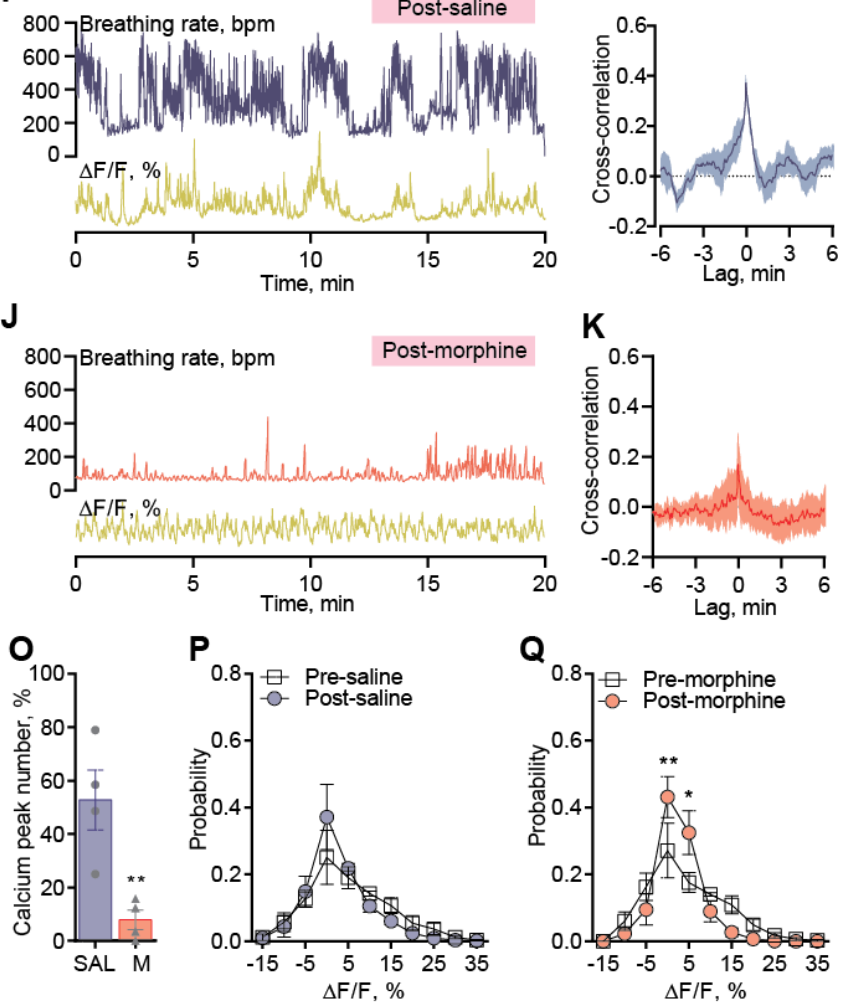

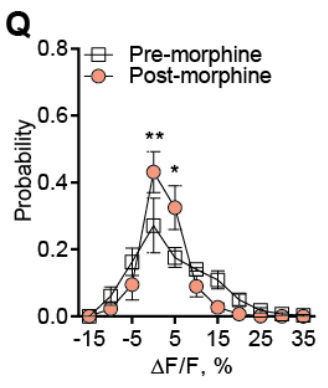

Figure 2. Morphine disrupts the tight correlation between $\mathrm{PBL}^{\text {oprmI }}$ neuronal activity and respiratory rate. (A) Schematics of simultaneous recording of respiration and $\mathrm{PBL}^{\mathrm{Oprm} 1}$ calcium activity with a thermistor sensor (implanted in the nasal cavity) and fiber photometry. (B) The genetically encoded calcium indicator, jGCaMP7s, was specifically expressed in the PBL ${ }^{O p r m l}$ neurons by stereotaxic injection of AAV-DIO-jGCaMP7s into the PBL of the $\mathrm{Oprml}^{\mathrm{Cre} /+}$ mice. (C) Time-matched traces of $\mathrm{PBL}^{\mathrm{Oprml}}$ activity and breathing rate as calculated from the thermistor sensor. (D, F, H, J) Simultaneously recorded $\mathrm{PBL}^{\text {Oprml }}$ calcium activity and respiratory rate before and after systemic saline or morphine injection. (E, G, I, K) Cross-correlogram of calcium signal and respiratory rate in the pre-saline, post-saline, pre-morphine, and post-morphine groups $(n=4)$. (L) Morphine injection significantly depressed respiratory rate to around $50 \%$ of the baseline $(n=4)$, whereas saline injection $(n=4)$ did not affect the respiratory rate. (M-N) Normalized distribution of breathing rate before and after saline $(\mathbf{M})$ and morphine $(\mathbf{N})$ injections $(n=4)$. (O) The number of calcium peaks was significantly decreased after morphine injection $(n=4)$ compared to saline injection $(n=4)$. $(\mathbf{P}-\mathbf{Q})$ Normalized distribution of calcium signals before and after saline $(\mathbf{P})$ and morphine $(\mathbf{Q})$ injections $(\mathrm{n}=4)$. Data are presented as mean \pm SEM. Statistical tests, Unpaired t-test $(\mathbf{L}, \mathbf{O})$ or Two-way ANOVA with Bonferroni's multiple comparison post-hoc test $(\mathbf{M}, \mathbf{N}, \mathbf{P}, \mathbf{Q})$. ${ }^{*}, p<0.05 ; * *, p<0.01$; ****, $p<0.0001$. Scale bar, $200 \mu \mathrm{m}$. 
A

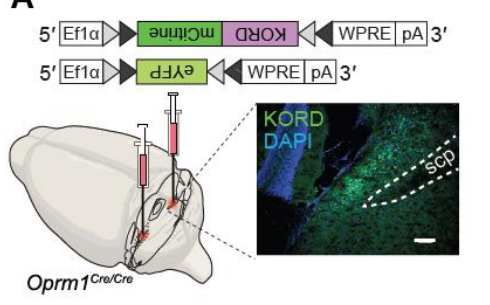

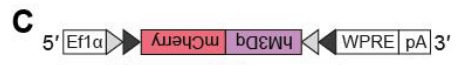

$5^{\prime}$ EFIa $\triangle$ d
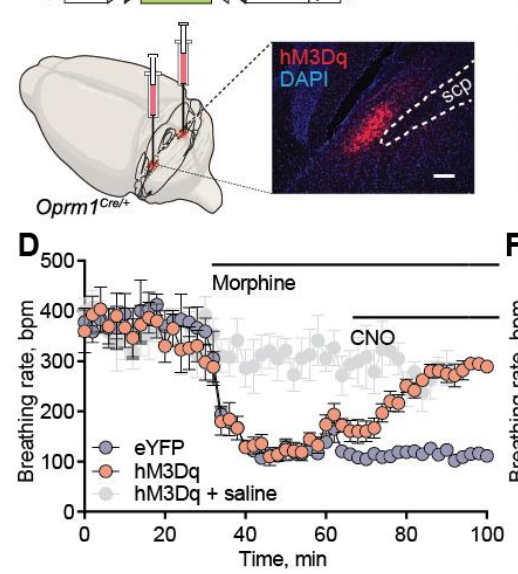

B

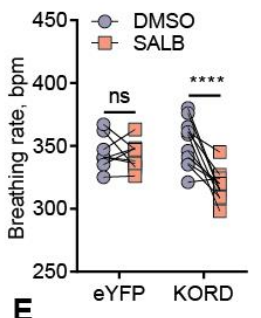

E

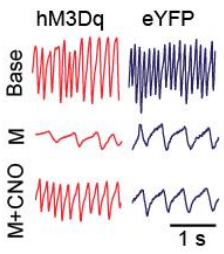

$\mathbf{F}_{500}$-O- Before CNO

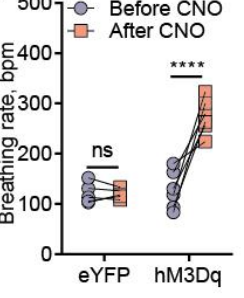

Figure 3. Inhibition of PBL ${ }^{\text {oprm } 1}$ neurons mimics OIRD, and chemogenetic activation of PBL ${ }^{\text {oprml }}$ neurons rescues OIRD. (A) Schematics of mimicking OIRD through $\mathrm{PBL}^{\text {oprml }}$ inhibition using an inhibitory $\kappa$-opioid receptor-derived DREADD (KORD). Here, AAV-DIO-KORD-mCitrine is stereotaxically injected into the bilateral PBL of $\mathrm{Oprm} 1^{\mathrm{Cre} / \mathrm{Cre}}$ mice to express in the PBL ${ }^{\text {Oprml }}$ neurons. (B) Salvinorin B (SALB, $7.5 \mathrm{mg} / \mathrm{kg}$ ) injection significantly decreased respiratory rate compared to the DMSO control in the KORD-expressing mice $(\mathrm{n}=11)$. In contrast, no changes were observed in eYFP-expressing mice $(n=8)$. (C) Schematics of rescuing OIRD through PBL $^{\text {Oprml }}$ activation by an excitatory DREADD, hM3Dq. Here, AAV-DIO-hM3Dq-mCherry was expressed in the bilateral PBL of $\mathrm{Oprml}^{\mathrm{Cre} / \mathrm{t}}$ mice. (D) Activation of $\mathrm{PBL}^{\mathrm{Oprml}}$ neurons by injecting Clozapine N-oxide (CNO, 5 $\mathrm{mg} / \mathrm{kg}$ ) rescued the morphine-induced respiratory depression to control levels in the hM3Dq-expressing group $(\mathrm{n}=$ 6), but not the eYFP-expressing group $(\mathrm{n}=5)$. ( $\mathbf{E - F})$ Representative plethysmograph and quantitative analysis depicting OIRD rescued by CNO injection in the hM3Dq-expressing group ( $\mathrm{n}=7)$, but not in the eYFP-expressing group $(\mathrm{n}=5)$. Statistical tests, Two-way ANOVA with Bonferroni's multiple comparison post-hoc test. ns, not significant; $* * * *, p<0.0001$. Scale bar, $200 \mu \mathrm{m}$. 
A
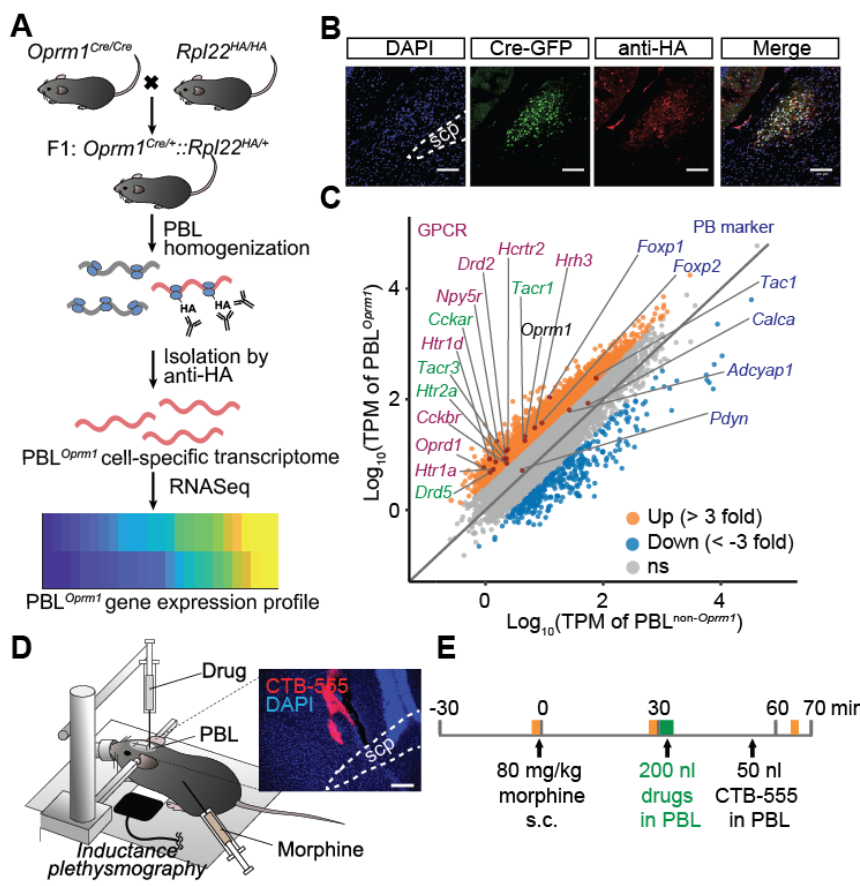

$\log _{10}($ TPM of PBL

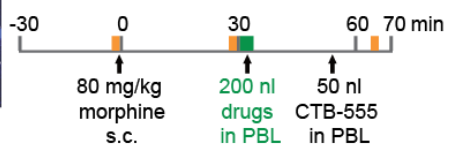

$\mathbf{F}_{1}$
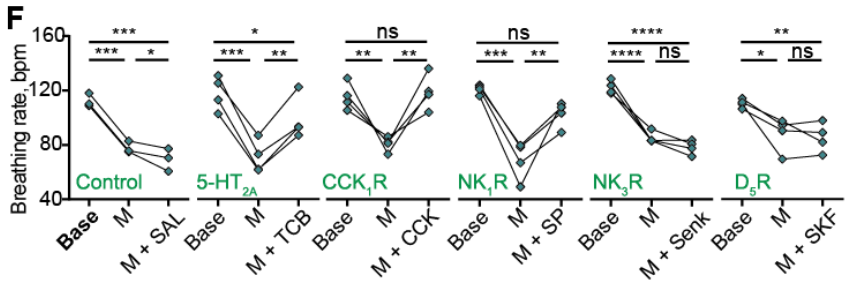

Figure 4. Rescue of OIRD through $\mathrm{PBL}^{\text {oprml }}$ stimulation via receptors identified by RiboTag RNA sequencing. (A) Schematics of obtaining $\mathrm{PBL}^{\text {Oprml }}$ active transcriptome. Oprm1 ${ }^{\mathrm{Cre} / \mathrm{Cre}}$ mice were crossed with RiboTag mice, which express hemagglutinin-tagged ribosomal protein L22 $\left(R p l 22^{H A}\right)$ in a Cre-dependent manner. After collecting the PBL, active mRNAs bound to the $R p l 22^{H A}$ were isolated with anti-HA antibody-mediated ribosome pull-down and sequenced. (B) Immunohistochemistry of the PBL region of the Oprm1 ${ }^{C r e}:: R p l 22^{H A}$ mice with anti-GFP and anti-HA antibodies showed that the Cre:GFP and Rpl22 ${ }^{\mathrm{HA}}$ were co-expressed in PBL ${ }^{\text {Oprm }}$ neurons. (C) Fourteen GPCR mRNAs (magenta and green) were enriched more than three-fold in PBL ${ }^{\text {oprml }}$ neurons compared with non-Oprm1 neurons in the PBL. Conventional parabrachial markers (dark blue), such as Foxp2, Tacl, and Adcyapl genes were enriched in PBL ${ }^{\text {Oprml }}$ neurons, but not Pdyn and Calca. (D-E) Schematics of rescuing OIRD through artificial stimulation of receptors expressed on $\mathrm{PBL}^{\text {Oprml }}$ neurons by PBL-specific injection of the agonists. Onset, histology of cholera toxin subunit B (CTB)-555 for marking the injection site. (F) Breathing rate decreased upon systemic morphine injection and rescued by PBL-specific injection of the agonists of 5-HT $2 \mathrm{~A}$, $\mathrm{CCK}_{1} \mathrm{R}$, and $\mathrm{NK}_{1} \mathrm{R}$. For the list of pharmacological agents, see Supplementary Table 1. Statistical tests, RM Oneway ANOVA with Tukey's multiple comparison post-hoc test, ns, not significant; *, $p<0.05$; **, $p<0.01$; ***, $p$ $<0.001, * * * *, p<0.0001$. Scale bar, $100 \mu \mathrm{m}(\mathbf{B})$ or $200 \mu \mathrm{m}(\mathbf{D})$. 


\section{Supplementary Materials}

\section{Materials and Methods}

Experimental Animals. The Oprm1 $1^{\text {Cre:GFP }}$ mouse line was generated from the lab of Dr. Richard Palmiter (see below). C57BL/6J (Stock No. 000664), Oprm flflfl (Stock No. 030074), RiboTag Rpl22 ${ }^{\text {HA/HA }}$ (Stock No. 011029) and Ai14 Gt(ROSA)26Sor tm14(CAG-tdTomato)Hze (Stock No. 007914) mouse lines were obtained from The Jackson Laboratory. Homozygous RiboTag and Ai14 mice were crossed with homozygous Oprml ${ }^{\text {Cre }}$ mice for RiboTag and anatomy experiments, respectively. Both male and female mice were used in all studies. Animals were randomized to experimental groups, and no sex differences were noted. Mice were maintained on a 12-h light/dark cycle and provided with food and water ad libitum.

Generation of Pprm1 $^{\mathrm{Cre}}$ mice. A cassette encoding Cre:GFP was inserted just 5' of the initiation codon in the first coding exon of the Oprml gene. The 5' arm ( 3.1 kb with PacI and SalI sites at 5' and 3' ends, respectively) and 3' arm ( $4.5 \mathrm{~kb}$ with $\mathrm{XhoI}$ and NotI sites at 5' and 3' ends, respectively) of Oprml gene were amplified from a C57BL/6 BAC clone by PCR using Q5 Polymerase (New England Biolabs, USA) and cloned into polylinkers of a targeting construct that contained mnCre:GFP, a FRT-flanked Sv40Neo gene for positive selection, and HSV thymidine kinase and $P g k$-diphtheria toxin A chain genes for negative selection. The mnCre:GFP cassette has a Myc-tag and nuclear localization signals at the N-terminus of Cre recombinase, which is fused to green fluorescent protein followed by a SV40 polyadenylation sequence. The construct was electroporated into G4 ES cells (C57BL/6 $\times 129 \mathrm{~Sv}$ hybrid), and correct targeting was determined by Southern blot of DNA digested with KpnI using a ${ }^{32} \mathrm{P}$-labeled probe downstream of the $3^{\prime}$ arm of the targeting construct. Twelve of the 84 clones analyzed were correctly targeted. One clone that was injected into blastocysts resulted in good chimeras that transmitted the targeted allele through the germline. Progeny were bred with Gt(Rosa)26Sor-FLP recombinase mice to remove the SV-Neo gene. Mice were then continuously backcrossed to C57BL/6 mice. Routine genotyping is performed with three primers: 5' CCT TCC ACT CAG AGA GTG GCG (Oprml forward), 5' CCT TCC ACT CAG AGA GTG GCG (Oprml reverse), and 5' GGC AAA TTT TGG TGT ACG GTC AG (Cre reverse). The wild-type allele gives a band of $\sim 500 \mathrm{bp}$, while the targeted allele provides a band with $\sim 400$ bp after 34 cycles with 20 -s annealing at 60 ${ }^{\circ} \mathrm{C}$.

Respiratory measurements. Inductance plethysmography. Inductance plethysmography was performed by placing a piezoelectric film beneath the chest of an anesthetized animal, which converts the chest movement into a voltage signal. The PowerLab system with LabChart 8 software (ADInstruments Inc., USA) was used for data acquisition, inspiratory and expiratory peak detection, and rate and amplitude calculation. Data were sampled at $100 \mathrm{or} 400 \mathrm{~Hz}$, low-pass filtered at $10 \mathrm{~Hz}$, and smoothed with a 100-ms moving window. Automatic peak detection was validated 
with manual peak detection. Since the location of the sensor is subject to the slight movements of the animal's body, the raw voltage value of the respiratory peak is less representative of the breathing amplitude.

Whole-body plethysmography (WBP). A custom-built WBP chamber was utilized for measuring respiratory changes. The PowerLab system with LabChart 8 software was used for data acquisition, inspiratory and expiratory peak detection, and rate and amplitude calculation. Data were sampled at $1 \mathrm{kHz}$, band-pass filtered at $1-10 \mathrm{~Hz}$, and smoothed with a 100-ms moving window. Automatic peak detection was validated with manual peak detection.

Mice were introduced into the WBP chamber for three 20 min habituation sessions before testing. Mice were kept in the chamber for 10 - 12 min during the testing session before and after the drug injection. After 5 - 10 min of chamber introduction, a stable pattern was reached, and the averaged value of a stabilized 1 min segment was analyzed.

Micro thermistor-based plethysmography. A custom-built micro thermistor was implanted into the mouse nasal cavity to detect changes in temperature between inspiratory and expiratory airflow (18). The sensor was assembled using a Negative Temperature Coefficient (NTC) thermistor (TE Connectivity Ltd., Switzerland), an interconnector (Mill-Max Mfg. Corp., USA), and a voltage divider (Phidgets Inc., Canada). PowerLab was used for data acquisition, inspiratory and expiratory peak detection, and rate and amplitude calculation. Data were sampled at 1 $\mathrm{kHz}$, filtered with a $0.4-25 \mathrm{~Hz}$ band-pass filter, and smoothed with a 50-ms moving window. Automatic peak detection was validated with manual peak detection.

Minute volume is approximated by first calculating the integral of the voltage channel for each breath, summing across one minute, then normalizing against the average value of the first 30 minutes. Values are shown after normalization as the raw values vary considerably across animals. For comparison before and after CNO injection, the averaged minute volume for 0-30 min and 30-75 min after morphine injection are used. Values during the episodes when the sensor accidentally fell off due to the animal body rotation were excluded.

Stereotaxic surgery. Mice were anesthetized with isoflurane (5\% induction, 1.5-2\% maintenance with a nose cone; Dräger Vapor 2000, Draegar, Inc., USA) and placed onto a water recirculating heating pad throughout the surgery. Mice were placed on a stereotaxic frame (David Kopf Instruments, USA), the skull was exposed, and the cranium was drilled with a micro motor handpiece drill (Foredom, USA: one or two holes for viral injection(s) or pharmacological delivery, two holes for screws with implantation, one or two holes for optic fibers, and one hole for a micro thermistor). The virus or drug was injected unilaterally (right side) or bilaterally into the PBL (anteroposterior (AP), -1 mm from lambda; mediolateral (ML), $\pm 1.5 \mathrm{~mm}$; dorsoventral (DV), $-3.5 \mathrm{~mm}$, re-zero at the midline with the same AP). Injection of the virus or drug was administered with a glass pipette (tips broken for an inner diameter of $20 \mu \mathrm{m}$ ) connected to the Nanoject III Programmable Nanoliter Injector (Drummond Scientific, USA) at a rate of $60 \mathrm{~nL} / \mathrm{min}$. Naloxone was injected at a rate of $100 \mathrm{~nL} / \mathrm{min}$ with a syringe (65458-01, Hamilton, 
USA) connected to an ultra-micropump (UMP-3, World Precision Instruments, USA). A glass pipette or syringe needle was retracted from the brain slowly after $5-10 \mathrm{~min}$. For implantation, optic fibers were implanted above the injection site with the DV noted below, and the micro thermistor head was carefully lowered into the hole above the nasal cavity (AP +3.5 from the nasal fissure, ML 0.3). The implants were covered with superglue and dental cement for stabilization. Behavioral experiments were performed three weeks after virus injection and one week after the micro-thermistor implantation unless otherwise noted.

For the PBL-specific conditional knockout of the Oprml gene, Oprm $1^{f l f l}$ mice were bilaterally injected with $200 \mathrm{~nL}$ of AAVDJ-CAG-Cre-GFP (1.25E+12 GC/mL) or control AAVDJ-CAG-GFP (2.10E+12 GC/mL) (Salk Institute Viral Vector Core) into the PBL.

For PBL-specific rescue of the Oprm1 gene, $\mathrm{Oprm}^{\mathrm{Cre} / \mathrm{Cre}}$ mice were bilaterally injected with $200 \mathrm{~nL}$ of AAV-hSynDIO-mCherry-T2A-FLAG-hOprm1 $(1.5 \mathrm{E}+13 \mathrm{GC} / \mathrm{mL})$ or control AAV-DIO-eYFP $(2.12 \mathrm{E}+12 \mathrm{GC} / \mathrm{mL})$ into the PBL.

For fiber photometry, $\mathrm{Oprml}^{\mathrm{Cre} / \mathrm{H}}$ mice were unilaterally injected with $200 \mathrm{~nL}$ of AAV-DIO-jGCaMP7s (3.75 E+13 $\mathrm{GC} / \mathrm{mL})$ or control AAV-DIO-eYFP $(2.12 \mathrm{E}+12 \mathrm{GC} / \mathrm{mL})$ into the PBL, and a stainless steel mono fiberoptic cannula (400 $\mu \mathrm{m}$ diameter, $0.37 \mathrm{NA}$, Doric Lenses) was implanted $0.05 \mathrm{~mm}$ above the injection site.

For chemogenetics, $200 \mathrm{~nL}$ of AAV-DIO-hM3Dq-mCherry $(6.56 \mathrm{E}+11 \mathrm{GC} / \mathrm{mL})$, AAV-DIO-KORD-mCitrine, or control AAV-DIO-eYFP $\left(2.12 \mathrm{E}+12 \mathrm{GC} / \mathrm{mL}\right.$ ) was bilaterally injected into the PBL of Oprm1 ${ }^{\mathrm{Cre} /+}$ (for hM3Dq) or $\mathrm{Oprml}^{\mathrm{Cre} / \mathrm{Cre}}$ (for KORD) mice.

Pharmacology. Morphine sulfate (Spectrum Chemical, USA) was dissolved in $0.9 \%$ saline to make a $4 \mathrm{mg} / \mathrm{mL}$ working concentration. The final concentration of morphine was $10 \mathrm{mg} / \mathrm{kg}$ in PBL-specific Oprml knockout tests, $80 \mathrm{mg} / \mathrm{kg}$ in PBL-specific rescue of OIRD, and $40 \mathrm{mg} / \mathrm{kg}$ in all other experiments. For the characterization of morphine-induced respiratory depression, mice were introduced into the WBP chamber 30 - 40 min after morphine injection.

For PBL-specific rescue of OIRD with naloxone, wild-type mice were kept under isoflurane anesthesia until the breathing rate was stable for $10 \mathrm{~min}$. Then, $80 \mathrm{mg} / \mathrm{kg}$ morphine (s.c.) was systemically administered. After $30 \mathrm{~min}$, $200 \mathrm{~nL}$ of $0.4 \mathrm{mg} / \mathrm{mL}$ naloxone (Somerset Therapeutics, USA) and FluoSpheres (540/560, 10\% v/v, Thermo Fisher, USA) mixture or a control $0.9 \%$ saline-FluoSpheres mixture was stereotaxically injected bilaterally into the PBL. Breathing was analyzed from three 2-min episodes: immediately before morphine injection, 30 min after morphine injection, and 10 min after naloxone or saline injection.

For endogenous receptor activation of $\mathrm{PBL}^{\mathrm{Oprm} l}$ neurons, wild-type mice were kept under 1-1.5\% isoflurane anesthesia until breathing rate is stable (fluctuation $<10 \mathrm{bpm}$ ) for $10 \mathrm{~min}$. Then, $80 \mathrm{mg} / \mathrm{kg}$ morphine (s.c.) was 
systemically administered. After $30 \mathrm{~min}, 200 \mathrm{~nL}$ of the pharmacological agent (Supplementary Table 1) was stereotaxically injected into the PBL bilaterally. $50 \mathrm{~nL}$ of Cholera Toxin Subunit B (CTB)-555 was subsequently injected with the same coordinates to mark the injection site. Breathing was analyzed from three 2-min episodes: immediately before morphine injection, $30 \mathrm{~min}$ after morphine injection, and $30 \mathrm{~min}$ after drug injection.

Fiber photometry. Fiber photometry (1-site Fiber Photometry System, 405 and 465 nm, Doric Lenses Inc, Canada) with Doric Neuroscience Studio software was used to record PBL ${ }^{\text {Oprm }}$ neural activities. GCaMP isosbestic fluorescence (405-nm excitation) and calcium-dependent fluorescence (465-nm excitation) were recorded at a sampling rate of $12 \mathrm{kHz}$, and data were analyzed with the Doric Neuroscience Studio software. F0 was calculated by a least mean squares fitting of the $405-\mathrm{nm}$ channel relative to the $465-\mathrm{nm}$ channel, and $\Delta \mathrm{F} / \mathrm{F}$ was calculated as $\left(\mathrm{F}_{465}-\mathrm{F}_{405}{ }_{\text {fitted }}\right) / \mathrm{F}_{405}$ fitted. Data were further analyzed with custom MATLAB scripts.

For concurrent measurements of $\mathrm{PBL}^{\text {Oprml }}$ neural activity and respiration, animals were recorded in their home cage for $30 \mathrm{~min}$ before and $30 \mathrm{~min}$ after intraperitoneal (i.p.) morphine injection at $40 \mathrm{mg} / \mathrm{kg}$. Cross-correlation analysis between the calcium signals and respiration data was performed with the z-scored data, then with the MATLAB "xcorr" function using the normalized option such that autocorrelations at zero lag equal 1. Calcium peaks were detected with the MATLAB "findpeaks" function.

Convergent cross-mapping. State-space reconstruction models were generated using the framework of convergent cross mapping (34), a nonlinear time series embedding method (53) based on the Takens theorem and its generalized form (54) that builds low-dimensional manifolds from time series and makes predictions across variables. Analysis and predictions were calculated using the R package rEDM 0.7.2 (https://cran.r-project.org/web/packages/rEDM/) for evaluation and rEDM 0.7.4 (https://ha0ye.github.io/rEDM/) for model predictions in the RStudio environment. This program was run on a dual Intel Xeon Gold 6148 Server with 384GB RAM or an Intel Core i9 $2.4 \mathrm{GHz}$ MacBook Pro with 32 GB RAM. Key parameters were determined individually by lagged coordinate embedding using the simplex function implementation in rEDM to optimize predictive skill as measured by the predicted over observed rho. Parameters include the delay tau, which gives the characteristic timescale of the time series, and the embedding dimensionality, which estimates the number of variables driving the system and approximates the real number of variables as given by the Whitney embedding theorem (55) as minimally equal to the real number $\mathrm{n}$ of variables, but no more than two times $n+1(n \leq E \leq 2 n+1)$. The choice of tau was also informed by minimizing mutual information (56). This approximately corresponds to an autocorrelation of $\sim 0.3$, which was applied if it maximized predictive skill across datasets. To prevent data contamination, an exclusion radius was applied that was larger than the respiration rate smoothing window of five timesteps. Whenever the data allowed, an exclusion radius of $\mathrm{E}^{*}$ tau was applied, unless the data was insufficient to apply this upper bound. In this case, the exclusion radius would be made just larger than tau. 
Chemogenetics. For KORD-mediated inhibition, salvinorin B (SALB, Cayman Chemical, USA) was diluted in $100 \%$ DMSO to make a $15 \mathrm{mg} / \mathrm{mL}$ stock solution, then further diluted in $0.9 \%$ saline $(10 \% \mathrm{v} / \mathrm{v})$ to make a 1.5 $\mathrm{mg} / \mathrm{mL}$ working suspension. The control used consisted of DMSO diluted in $0.9 \%$ saline to make $10 \% \mathrm{v} / \mathrm{v}$. Mice were injected with $5 \mu \mathrm{L} / \mathrm{g}$ of body weight. The final concentration was $7.5 \mathrm{mg} / \mathrm{kg}$. Behavioral testing was performed immediately after the SALB injection. Animals that did not show the viral expression on one side were excluded from the analysis.

For hM3Dq-mediated excitation, clozapine-N-oxide (CNO, Cayman Chemical, USA) was diluted in $0.9 \%$ saline to make a $1 \mathrm{mg} / \mathrm{mL}$ working solution. Mice were injected with $5 \mu \mathrm{L} / \mathrm{g}$ of body weight, and the final concentration was $5 \mathrm{mg} / \mathrm{kg}$. For quantification of the CNO effect, both the $0-10 \mathrm{~min}$ before and $20-30 \mathrm{~min}$ after CNO injection periods were analyzed.

RiboTag profiling and library generation. Isolation of polysome-associated mRNA using RiboTag was performed as previously described with minor modification $(37,57)$. Briefly, 250- $\mu \mathrm{m}$ thick slices containing the PBL were obtained using a VT 1200S Vibratome (Leica, Germany). The region of interest was further dissected with surgical scissors. Tissues were transferred into $1.5 \mathrm{~mL}$ microcentrifuge tubes containing homogenization buffer (50 mM Tris, pH 7.5, $100 \mathrm{mM} \mathrm{KCl,} 12 \mathrm{mM} \mathrm{MgCl}$, 1\% Nonidet P-40, $1 \mathrm{mM}$ dithiothreitol (DTT), $200 \mathrm{U} / \mathrm{mL}$ RNasin, $1 \mathrm{mg} / \mathrm{mL}$ heparin, $100 \mu \mathrm{g} / \mathrm{mL}$ cycloheximide, and $1 \times$ protease inhibitor mixture) and mechanically dissociated and lysed using pellet pestles (7495211500-DS, DWK Life Sciences LLC, USA). Lysates were centrifugated for $10 \mathrm{~min}$ at $12,000 \mathrm{rpm}$ at $4{ }^{\circ} \mathrm{C}$. Post mitochondrial supernatants were transferred to fresh $1.5 \mathrm{~mL}$ microcentrifuge tubes. For immunoprecipitation, $4 \mu \mathrm{L}$ of anti-hemagglutinin1.1 antibody (BioLegend, USA) was added to the lysate-containing tube and incubated for $4 \mathrm{~h}$ at $4{ }^{\circ} \mathrm{C}$ on a microtube rotator. After incubation, magnetic protein A/G beads (Cat. No. 88803; Thermo Fisher Scientific) were added to the lysate with antibody prior to incubation on a microtube rotator at $4{ }^{\circ} \mathrm{C}$ overnight. The following day, the microcentrifuge tubes were placed into the magnetic stand on ice. The supernatant was removed from sample tubes and used for non-Oprm1 control subsequently. The magnetic beads were washed with high-salt buffer $(50 \mathrm{mM}$ Tris, $\mathrm{pH} 7.5,300 \mathrm{mM} \mathrm{KCl}, 12 \mathrm{mM}$ $\mathrm{MgCl}_{2}, 1 \%$ Nonidet P-40, $1 \mathrm{mM}$ DTT, and $100 \mu \mathrm{g} / \mathrm{mL}$ cycloheximide) to remove the non-specific binding from immunoprecipitation. After washing, $350 \mu \mathrm{L}$ of RLT plus buffer with $\beta$-mercaptoethanol from the RNeasy Mini Kit (Qiagen, Germany) was added. The extraction of total RNA was performed with the RNeasy Mini Kit. All RNA samples were quantified with the Qubit RNA Assay Kit (Invitrogen, USA) and analyzed with the RNA 6000 Pico Kit (Agilent, USA).

Isolated RNA was prepared using the Trio RNA-Seq (Cat. No. 0507-08; NuGEN, USA). Briefly, cDNA was synthesized from the total RNA using reverse transcriptase with oligo dT and resynthesized to produce doublestranded cDNA. After amplification of double-stranded cDNA, cDNA was purified with AMPure XP Beads (Cat. 
612

No. A63881; Beckman Coulter, USA), fragmented to the library, and classified using a barcoded adaptor. All libraries were quantified by qPCR and analyzed with the RNA 6000 Pico Kit.

RiboTag profiling analysis. RNA library quality was confirmed using the 2100 Bioanalyzer (Agilent, USA). Barcoded samples were pooled and sequenced on the NextSeq500 (Illumina, USA) with the 75-bp read length single-end format. Image analysis and base calling were conducted using the Illumina CASAVA-1.8.2 software. Sequencing read quality was evaluated with the FastQC package (http://www.bioinformatics.babraham.ac.uk/projects/fastqc). Fastq reads were aligned to the reference genome (GRCm38.p6) using the STAR tool (version 2.7.2) (58). The quantification package RSEM (version 1.2.28) (59) was utilized to calculate gene expression from BAM files. In doing so, estimated count and TPM (Transcripts Per Million) were computed. Fold changes were calculated from TPM values (estimated counts > 20) between HA-tag and controls. To visualize fold changes, we utilized the ggplot 2 package from R. UP (> 3 fold change) and DOWN ( $<-3$ fold change) were highlighted with orange and blue colors, respectively. Key genes for the parabrachial marker and GPCRs were further annotated.

Histology. Mice were euthanized with $\mathrm{CO}_{2}$ at a flow rate of 1.2 liters per minute (LPM), perfused intracardially with ice-cold phosphate-buffered saline (PBS), and fixed with $4 \%$ paraformaldehyde (PFA) in phosphate buffer (PB). The brain was extracted, post-fixed in $4 \%$ PFA overnight and dehydrated in $30 \%$ sucrose in PBS until sliced. Frozen brains were cut into $50 \mu \mathrm{m}$ coronal slices using a CM 1950 cryostat (Leica, Germany) and stored in PBS before mounting. The slices were mounted on Superfrost Microscope Slides (Fisher Scientific, USA) with DAPI Fluoromount-G mounting media (Southern Biotech, USA) for imaging.

Immunohistochemistry. To validate the co-expression of Cre:GFP and Rpl22:HA in PBL ${ }^{\text {oprml }}$ neurons, immunohistochemistry for HA and GFP was performed. To validate the conditional knockout of the Oprml gene in the PBL, we performed immunohistochemistry for MOR.

Mice were euthanized with $\mathrm{CO}_{2}$ at a flow rate of 1.2 LPM, perfused intracardially with ice-cold PBS, and fixed with $4 \%$ PFA in PB. The brain was extracted, post-fixed in $4 \%$ PFA overnight and dehydrated in $30 \%$ sucrose in PBS until sliced. Frozen brains were cut into $30 \mu \mathrm{m}$ coronal slices with a CM 1950 cryostat and stored in PBS.

Sections were washed with PBST (PBS with 3\% Tween-20, Fisher BioReagents, USA). After blocking with $3 \%$ normal donkey serum (NDS, Jackson ImmunoResearch Inc., USA) for $1 \mathrm{~h}$ at room temperature and rinsing with PBST, the slices were incubated with the corresponding antibodies: mouse monoclonal anti-HA1.1 (1:1000, BioLegend, USA), chicken anti-GFP (1:1000, Aves Labs Inc, USA), and rabbit anti-MOR (1:1000, ImmunoStar, USA) antibodies at $4{ }^{\circ} \mathrm{C}$ for overnight (anti-HA1.1 and anti-GFP) or $24 \mathrm{~h}$ (anti-MOR). The next day, brain tissues were rinsed with PBST and incubated in Alexa Fluor ${ }^{\circledR}$ 647-conjugated Donkey Anti-Mouse IgG, Alexa Fluor ${ }^{\circledR} 488$ conjugated Donkey Anti-Chicken IgY, and Alexa Fluor ${ }^{\circledR}$ 647-conjugated Donkey Anti-Rabbit IgG (1:1000, Jackson 
ImmunoResearch Inc., USA) in $3 \%$ NDS for $90 \mathrm{~min}$ at room temperature. After washing with PBS, brain slices were mounted on Superfrost Microscope Slides with DAPI Fluoromount-G mounting media for imaging.

RNA in situ hybridization. RNA in situ hybridization was performed using the RNAscope Fluorescent Multiplex Assay using the probes and kits purchased from Advanced Cell Diagnostics (ACD, USA). Brains were collected from wild type mice and immediately frozen with 2-methylbutane chilled with dry ice. Frozen brains were cut into 20- $\mu$ m coronal slices with a CM 1950 cryostat and directly mounted onto the Superfrost Plus Microscope Slides (Fisher Scientific). Sample preparation, pretreatment, and signal detection were performed according to the ACD protocols. Probes used are listed below: Oprm1 (ACD \#315841), Htr2a (\#401291), Cckar (\#313751), Drd5 (\#494411), Tacr3 (\#481671), Tacr1 (\#428781), Cre (\#402551), Slc17a6 (\#319171), and Slc32al (\#319191).

Two to three representative images from the PBL ( $\mathrm{AP}=-5.0$ to -5.2$)$ were selected from $\mathrm{n}=3$ mice, and PBL cells within a field of view of $300 \mu \mathrm{m}$ x $300 \mu \mathrm{m}$ (for Oprm1 colocalization with Cre, Htr2a, Cckar, Tacr3, Tacrl, and Drd5) or $600 \mu \mathrm{m} \times 600 \mu \mathrm{m}$ (for Oprm1 colocalization with Slc17a6 and Slc32al) were used for quantification. Quantification of the colocalization is done manually with ImageJ software according to the ACDBio technical note. DAPI-stained nuclei were first identified, then the cell contour was defined with a $2-\mu \mathrm{m}$ radius surrounding the DAPI signals. Cells containing at least five puncta inside the imaginary boundary were labeled as positive.

Imaging. Images for verification of injection and implantation site and anti-MOR staining for conditional knockout validation were taken with a BZ-X710 all-in-one fluorescence microscope with BZ-X viewer software under a 10X, 0.45 NA objective (Keyence, Japan). Images for anti-HA \& anti-GFP immunostaining, RNAscope, and

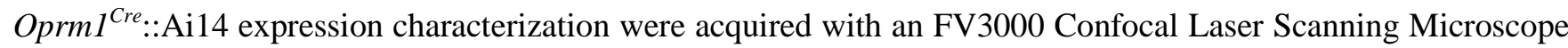
with FV31S-SW software under a 20X, 0.75 NA or 40X, 0.95 NA UPLSAPO objective (Olympus, Japan). For comparison, images were processed with the same gain, offset, and exposure time.

Statistical Analysis. All data are shown as mean \pm SEM and analyzed using either a student's t-test, one-way ANOVA with Tukey's post hoc comparison, or two-way ANOVA with Bonferroni's post hoc comparison. All statistical analyses were performed with Prism 6 (GraphPad Software Inc., USA). ns $p>0.05, * p<0.05$, ** $p<$ $0.01, * * * p<0.001, * * * * p<0.0001$.

For a detailed list of resources, please refer to Supplementary Table 2. 


\section{Supplementary Figures}

A
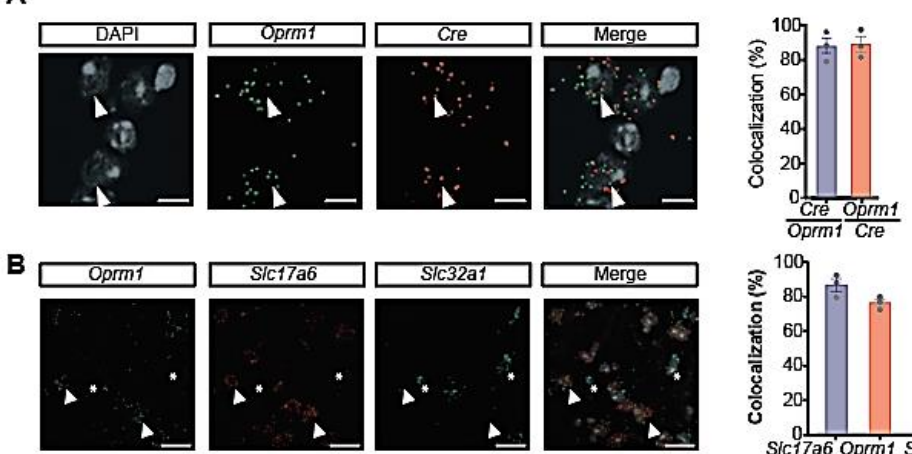

C
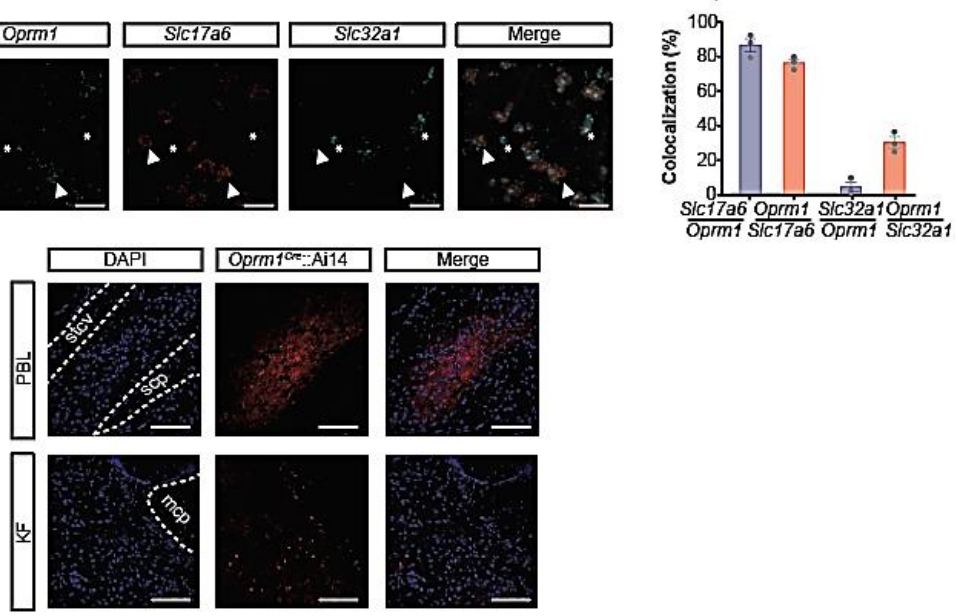

Supplementary Figure 1. Characterization of the $O p r m 1^{\text {Cre }}$ mouse line and PBL ${ }^{\text {Oprm }}$ neurons. (A) RNA in situ hybridization and quantification of the colocalization for Oprm1 and Cre mRNAs in the PBL of Oprm1 ${ }^{\text {Crelt }}$ mice ( $\mathrm{n}=902$ cells from 3 animals). Arrowheads, double-labeled cells. Scale bar, $10 \mu \mathrm{m}$. (B) RNA in situ hybridization and quantification of the colocalization for Oprm1, Slc17a6 (encoding VGLUT2), and Slc32al (encoding VGAT) mRNAs in the PBL of wild type mice $\left(\mathrm{n}=1552\right.$ and 1431 cells from 3 animals). Arrowheads, Oprm1 $^{+} /$Slcl a $^{+}$ cells; asterisks, Oprm1 ${ }^{-} /$Slc32al ${ }^{+}$cells. Scale bar, $50 \mu \mathrm{m}$. (C) Example histology from Oprm1 $^{\mathrm{Cre}}::$ Ai14 double transgenic mice showing tdTomato expression in the PBL and KF under the same microscope settings. Anatomical landmarks for PBL: stcv, ventral spinocerebellar tract; scp, superior cerebellar peduncle; mcp, middle cerebellar peduncle. Scale bar, $200 \mu \mathrm{m}$. 


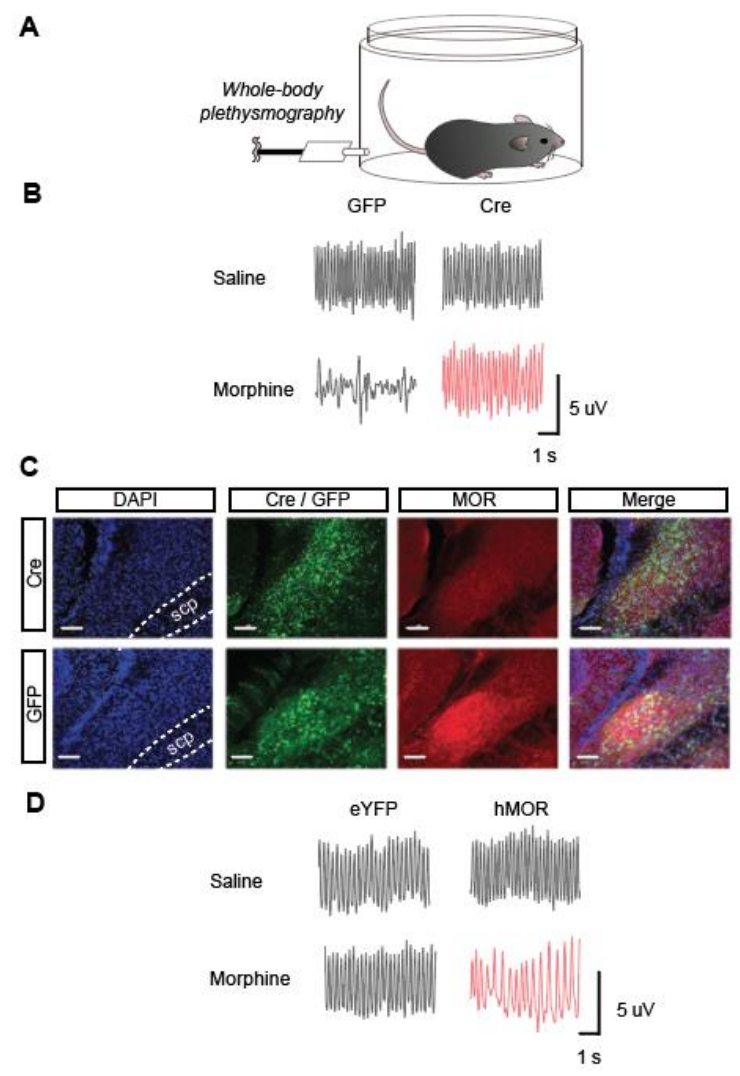

682 Supplementary Figure 2. MOR signaling in the PBL is indispensable for OIRD. (A) Whole-body 683 plethysmography measured respiratory parameters before and after systemic injection of saline and morphine, after 684 conditional ablation (B) and rescue (D) of the MOR signaling in the PBL. (B) Example plethysmograph after saline 685 and morphine injections into the Oprm1 $1^{f l f l}$ mice expressing AAV-GFP and AAV-Cre-GFP in the PBL. (C) 686 Confirmation of MOR deletion by MOR immunohistochemistry after stereotaxic injection of AAV-Cre-GFP and 687 control AAV-GFP into the PBL of the Oprm $^{f l / f l}$ mice. Scale bar, $100 \mu \mathrm{m}$. (D) Example plethysmograph after saline 688 and morphine injections into the $\mathrm{Oprm}^{\mathrm{Cre} / \mathrm{Cre}}$ mice expressing AAV-DIO-eYFP and AAV-DIO-hMOR in the PBL. 
A

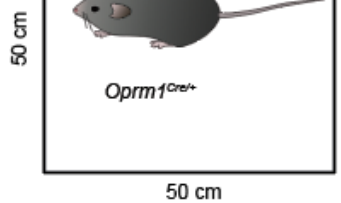

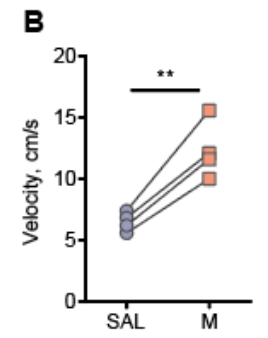

691 Supplementary Figure 3. Morphine effect on locomotor sensitization. (A) Locomotor activity of $\mathrm{Oprm}^{\mathrm{Cre} / \mathrm{+}} \mathrm{mice}$ 692 after morphine $(40 \mathrm{mg} / \mathrm{kg}$, i.p.) or saline injection was measured in the open field. (B) Systemic morphine injection 693 increased locomotor activity in $\mathrm{pprml}^{\mathrm{Cr} / \mathrm{t}}$ mice. Paired t-test, $* *, \quad p<0.01$. 694 695 696 
A

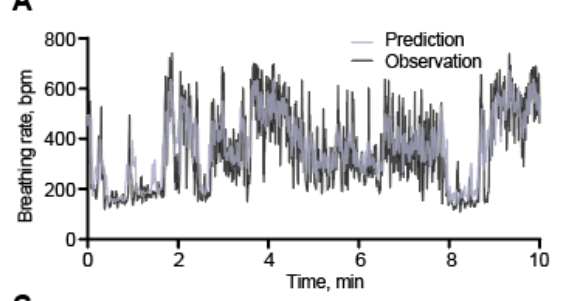

C

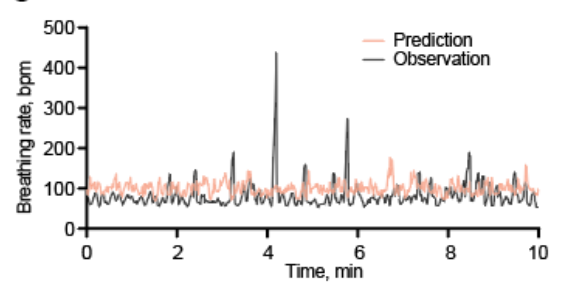

B 15.5

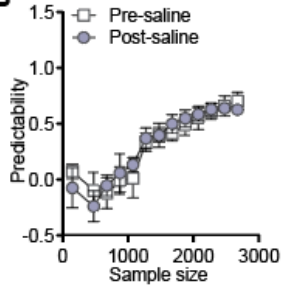

D 1.5 . Pre-morphine

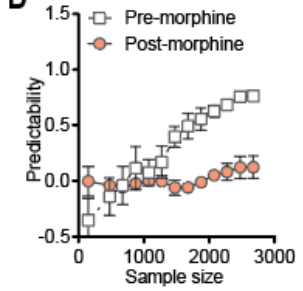

Supplementary Figure 4. Morphine eliminated the breathing predictor. Convergent cross-mapping (CCM) prediction of breathing rate using calcium activity as inputs before and after saline (0.9\%, i.p., A and B) and morphine (40 mg/kg, i.p., C and D) injection. (A) After saline injection, the predicted and observed breathing rate traces followed closely with each other during the 10-min example. (B) Model predictability increased with the sample size before and after saline injection $(\mathrm{n}=4)$. (C) After morphine injection, the predicted and observed breathing rate traces no longer matched with each other. (D) Model predictability increased with the sample size before morphine injection, but the relationship was completely abolished after morphine injection $(\mathrm{n}=4)$. 

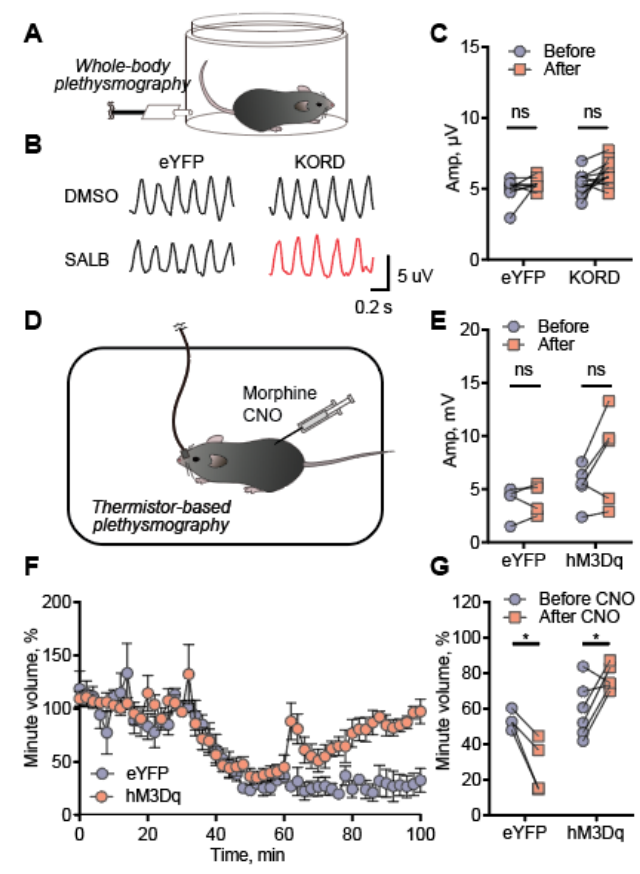

Supplementary Figure 5. (A) Whole-body plethysmography was used to measure respiratory parameters before and after systemic injection of DMSO and SALB in the Pprm $^{\text {Cre/Cre }}$ mice expressing AAV-DIO-eYFP and AAVDIO-KORD in PBL ${ }^{\text {oprml }}$ neurons. (B) Example plethysmograph after DMSO and $7.5 \mathrm{mg} / \mathrm{kg}$ SALB injections in eYFP and KORD groups. KORD-expressing animals displayed a slower respiratory rate after SALB injection compared to other groups. (C) Respiratory amplitude was not significantly changed before and after SALB injection, in both eYFP $(\mathrm{n}=8)$ and KORD $(\mathrm{n}=11)$-expressing animals. Two-way ANOVA with Bonferroni's multiple comparison post-hoc test, ns, not significant. (D) Thermistor-based plethysmography was used for measuring respiration before and after systemic injection of $40 \mathrm{mg} / \mathrm{kg}$ morphine and CNO in the $\mathrm{Oprm} 1^{\mathrm{Cre} / \mathrm{+}}$ mice expressing AAV-DIO-eYFP and AAV-DIO-hM3Dq in PBL ${ }^{\text {Oprm }}$ neurons. (E) Respiratory amplitude was not significantly changed before and after CNO injection, in both eYFP $(n=5)$ and hM3Dq-expressing animals $(n=$ 6). Although there was a trend of increase in the hM3Dq group, it failed to reach statistical significance. Two-way ANOVA with Bonferroni's multiple comparison post-hoc tests, ns, not significant. (F) Activation of PBL ${ }^{\text {oprm }}$ neurons by injecting CNO $(5 \mathrm{mg} / \mathrm{kg}$, i.p.) in the $\mathrm{hM} 3 \mathrm{Dq}$-expressing group $(\mathrm{n}=6)$ completely rescued the minute volume to baseline level after morphine-induced respiratory depression, but not in eYFP-expressing group $(n=5)$. (G) Quantitative analysis of $\mathbf{F}$ showing CNO injection significantly increased the minute volume in the hM3Dqexpressing group $(n=6)$, whereas failed to rescue in the eYFP-expressing group $(n=5)$. Two-way ANOVA with Bonferroni's multiple comparison post-hoc test. *, $p<0.05$. 


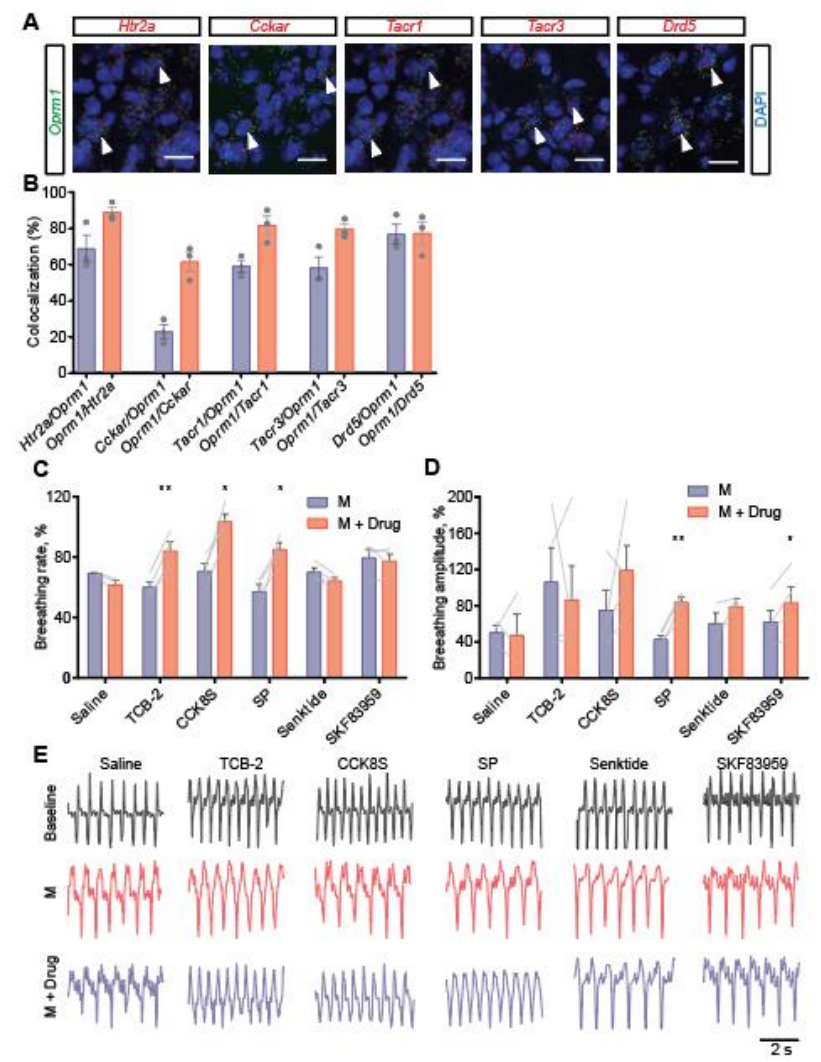

727 Supplementary Figure 6. Rescuing of OIRD by activating endogenous GPCRs expressed on PBL ${ }^{\text {oprml }}$ neurons. (A) RNA in situ hybridization confirms the co-expression of mRNA of Oprm1 and five selected GPCRs, Htr2a, Cckar, Tacr1, Tacr3, and Drd5, in the wild type mice. Arrowheads, double-labeled cells. Scale bar, $50 \mu \mathrm{m}$. (B) Quantification of RNA in situ hybridization showing the colocalization of Oprm1 and selective GPCR genes in the PBL of wild type mice (770, 539, 836, 657, 431 cells for Htr2a, Cckar, Tacr1, Tacr3, and Drd5, respectively). (CD) Normalized breathing rate (C) and amplitude (D) before morphine injection (Baseline), 30 minutes after morphine injection $(80 \mathrm{mg} / \mathrm{kg}$, s.c., $\mathrm{M})$, and 30 minutes after drug injection into the PBL $(\mathrm{M}+\mathrm{Drug})$, for all six drugs tested. Large variations in amplitude are due to the technical limitations of the piezoelectric sensor since its location is subjective to the slight movements of the animal's body. Paired t-test, ${ }^{*}, p<0.05 ; * *, p<0.01$. e Example plethysmograph showing the breathing rhythm changes at each stage of the experiment. Note the decreased breathing rate after morphine injection and increased breathing rate after TCB-2, CCK8S, and SP injections. The exact shape of the plethysmograph varies due to the placement of the piezoelectric sensor. 


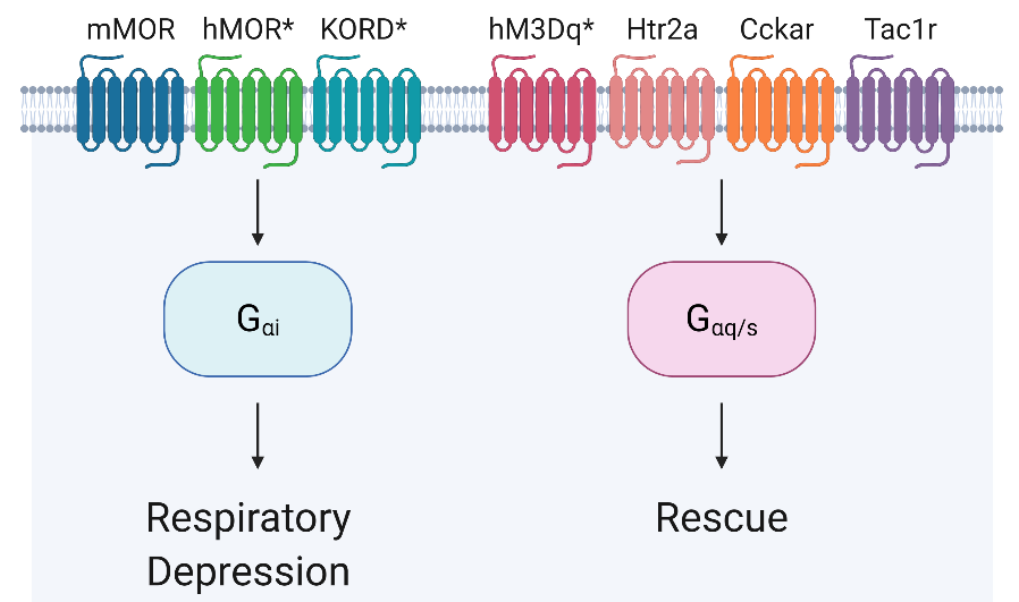

PBL ${ }^{\text {oprm1 }}$

741 Supplementary Figure 7. Summary of the current study. PBL ${ }^{\text {Oprml }}$ neurons are critical players in OIRD 742 pathogenesis and promising therapeutic targets for treating OIRD. In intact mice, inhibition of PBL ${ }^{\text {Oprm }}$ neurons 743 through $\mathrm{G}_{\mathrm{i}}$-coupled GPCRs via endogenous MOR (mMOR), human MOR (hMOR), and KOR-derived DREADD 744 (KORD) leads to respiratory depression. In contrast, activation of these neurons via artificial (hM3Dq) or 745 endogenous $\mathrm{G}_{\mathrm{q} / \mathrm{s}}$-coupled GPCRs (Htr2a, Cckar, Tac1r) rescues OIRD. Artificial GPCRs are marked with asterisks. 746 Created with BioRender.com. 
757 Supplementary Table 1. List of pharmacological agents used in the current study.

\begin{tabular}{|c|c|c|c|c|c|}
\hline Drug Name & $\begin{array}{l}\text { Receptor } \\
\text { (Gene) }\end{array}$ & $\begin{array}{l}\text { Receptor } \\
\text { Family }\end{array}$ & Concentration & Solvent & Company \\
\hline Naloxone & MOR (Oprml) & $\mathrm{Gi}$ & $0.4 \mathrm{mg} / \mathrm{mL}$ (i.c., intracranial) & $0.9 \%$ saline & $\begin{array}{l}\text { Somerset } \\
\text { Therapeutics }\end{array}$ \\
\hline Morphine & MOR (Oprml) & Gi & $\begin{array}{l}80 \mathrm{mg} / \mathrm{kg} \text { (s.c., for anesthesia } \\
\text { experiments); } \\
10 \mathrm{mg} / \mathrm{kg} \text { (i.p., for PBL-specific } \\
\text { Oprml knockout); } \\
40 \mathrm{mg} / \mathrm{kg} \text { (i.p., for all other } \\
\text { experiments) }\end{array}$ & $0.9 \%$ saline & $\begin{array}{l}\text { Spectrum } \\
\text { Chemical }\end{array}$ \\
\hline $\begin{array}{l}\text { Salvinorin } \mathrm{B} \\
(\mathrm{SALB})\end{array}$ & KORD & Gi & $7.5 \mathrm{mg} / \mathrm{kg}$ (i.p.) & $\begin{array}{l}0.9 \% \text { saline } \\
\text { containing } \\
10 \% \text { DMSO }\end{array}$ & $\begin{array}{l}\text { Cayman } \\
\text { Chemical }\end{array}$ \\
\hline $\begin{array}{l}\text { Clozapine-N- } \\
\text { oxide (CNO) }\end{array}$ & hM3Dq & $\mathrm{Gq}$ & $5 \mathrm{mg} / \mathrm{kg}$ (i.p.) & $0.9 \%$ saline & $\begin{array}{l}\text { Cayman } \\
\text { Chemical }\end{array}$ \\
\hline TCB-2 & $5-\mathrm{HT}_{2 \mathrm{~A}}(H t r 2 a)$ & $\mathrm{Gq}$ & $1 \mathrm{mg} / \mathrm{mL}$ (i.c.) & $0.9 \%$ saline & $\begin{array}{l}\text { Tocris } \\
\text { Bioscience }\end{array}$ \\
\hline $\begin{array}{l}\text { CCK } \\
\text { Octapeptide, } \\
\text { sulfated } \\
(\mathrm{CCK} 8 \mathrm{~S})\end{array}$ & $\mathrm{CCK}_{1} \mathrm{R}($ Cckar $)$ & $\mathrm{Gq}$ & $1 \mathrm{mg} / \mathrm{mL}$ (i.c.) & $0.9 \%$ saline & Abcam \\
\hline Substance P & $\mathrm{NK}_{1} \mathrm{R}($ Tacrl $)$ & Gq, Gs & $1 \mathrm{mg} / \mathrm{mL}$ (i.c.) & $0.9 \%$ saline & $\begin{array}{l}\text { Cayman } \\
\text { Chemical }\end{array}$ \\
\hline Senktide & $\mathrm{NK}_{3} \mathrm{R}($ Tacr3) & $\mathrm{Gq}$ & $1 \mathrm{mg} / \mathrm{mL}$ (i.c.) & $0.9 \%$ saline & $\begin{array}{l}\text { Cayman } \\
\text { Chemical }\end{array}$ \\
\hline SKF-83959 & $\mathrm{D}_{5} \mathrm{R}(\operatorname{Drd5})$ & Gs & $1 \mathrm{mg} / \mathrm{mL}$ (i.c.) & $\begin{array}{l}0.9 \% \quad \text { saline } \\
\text { containing } 5 \% \\
\text { DMSO }\end{array}$ & $\begin{array}{l}\text { Tocris } \\
\text { Bioscience }\end{array}$ \\
\hline
\end{tabular}




\begin{tabular}{|c|c|c|c|}
\hline Type & Designation & Source or reference & Identifiers \\
\hline \multirow[t]{4}{*}{ Mouse strains } & Oprm1-Cre:GFP & Laboratory of Dr. Richard Palmiter & N/A \\
\hline & Wild type C57BL/6J & Jackson Laboratory & $\begin{array}{ll}\text { Stock } & \text { No. } \\
000664 & \end{array}$ \\
\hline & RiboTag $R p l 22^{H A / H A}$ & Jackson Laboratory & $\begin{array}{ll}\text { Stock } & \text { No. } \\
011029 & \end{array}$ \\
\hline & Ai14 Gt(ROSA)26Sor ${ }^{t m 14(C A G-t d T o m a t o) H z e}$ & Jackson Laboratory & $\begin{array}{ll}\text { Stock } \quad \text { No. } \\
007914\end{array}$ \\
\hline \multirow[t]{7}{*}{ Virus } & AAVDJ-CAG-Cre-GFP & Salk Institute Viral Vector Core & N/A \\
\hline & AAVDJ-CAG-GFP & Salk Institute Viral Vector Core & N/A \\
\hline & $\begin{array}{l}\text { AAV-hSyn-DIO-mCherry-T2A- } \\
\text { FLAG-hOprm1 }\end{array}$ & Laboratory of Dr. Matthew Banghart & N/A \\
\hline & AAV1-syn-FLEX-jGCaMP7s-WPRE & Addgene & $\begin{array}{l}\text { Addgene\# } \\
104491\end{array}$ \\
\hline & $\begin{array}{l}\text { AAVDJ-EF1a-DIO-hM3D(Gq)- } \\
\text { mCherry }\end{array}$ & Salk Institute Viral Vector Core & $\begin{array}{l}\text { Addgene\# } \\
50460\end{array}$ \\
\hline & AAV-DIO-KORD-mCitrine & Laboratory of Dr. Richard Palmiter & N/A \\
\hline & AAV1-DIO-eYFP & Laboratory of Dr. Richard Palmiter & N/A \\
\hline \multirow[t]{6}{*}{ Antibodies } & Anti-hemagglutinin1.1, mouse $(1: 1000)$ & BioLegend & $\begin{array}{l}\text { RRID } \\
\text { AB_2565335 }\end{array}$ \\
\hline & Anti-GFP, chicken (1:1000) & Aves Labs & $\begin{array}{l}\text { RRID } \\
\text { AB_2307313 }\end{array}$ \\
\hline & Anti-MOR, rabbit (1:1000) & ImmunoStar & $\begin{array}{l}\text { RRID } \\
\text { AB_572251 }\end{array}$ \\
\hline & $\begin{array}{l}\text { Alexa Fluor® } 647 \text {-conjugated Donkey } \\
\text { Anti-Mouse IgG }(1: 1000)\end{array}$ & $\begin{array}{ll}\text { Jackson } & \text { ImmunoResearch } \\
\text { Laboratories } & \end{array}$ & $\begin{array}{l}\text { RRID } \\
\text { AB_2340863 }\end{array}$ \\
\hline & $\begin{array}{l}\text { Alexa Fluor }{ }^{\circledR} 488 \text {-conjugated Donkey } \\
\text { Anti-Chicken } \operatorname{IgY}(1: 1000)\end{array}$ & $\begin{array}{ll}\text { Jackson } & \text { ImmunoResearch } \\
\text { Laboratories } & \end{array}$ & $\begin{array}{l}\text { RRID } \\
\text { AB_2340375 }\end{array}$ \\
\hline & $\begin{array}{l}\text { Alexa Fluor }{ }^{\circledR} \text { 647-conjugated Donkey } \\
\text { Anti-Rabbit IgG }(1: 1000)\end{array}$ & $\begin{array}{ll}\text { Jackson } & \text { ImmunoResearch } \\
\text { Laboratories } & \end{array}$ & $\begin{array}{l}\text { RRID } \\
\text { AB_2492288 }\end{array}$ \\
\hline \multirow[t]{4}{*}{ RNAscope probes } & Oprm1 & Advanced Cell Diagnostics & 315841 \\
\hline & $\mathrm{Htr} 2 \mathrm{a}$ & Advanced Cell Diagnostics & 401291 \\
\hline & Cckar & Advanced Cell Diagnostics & 313751 \\
\hline & Drd5 & Advanced Cell Diagnostics & 494411 \\
\hline
\end{tabular}




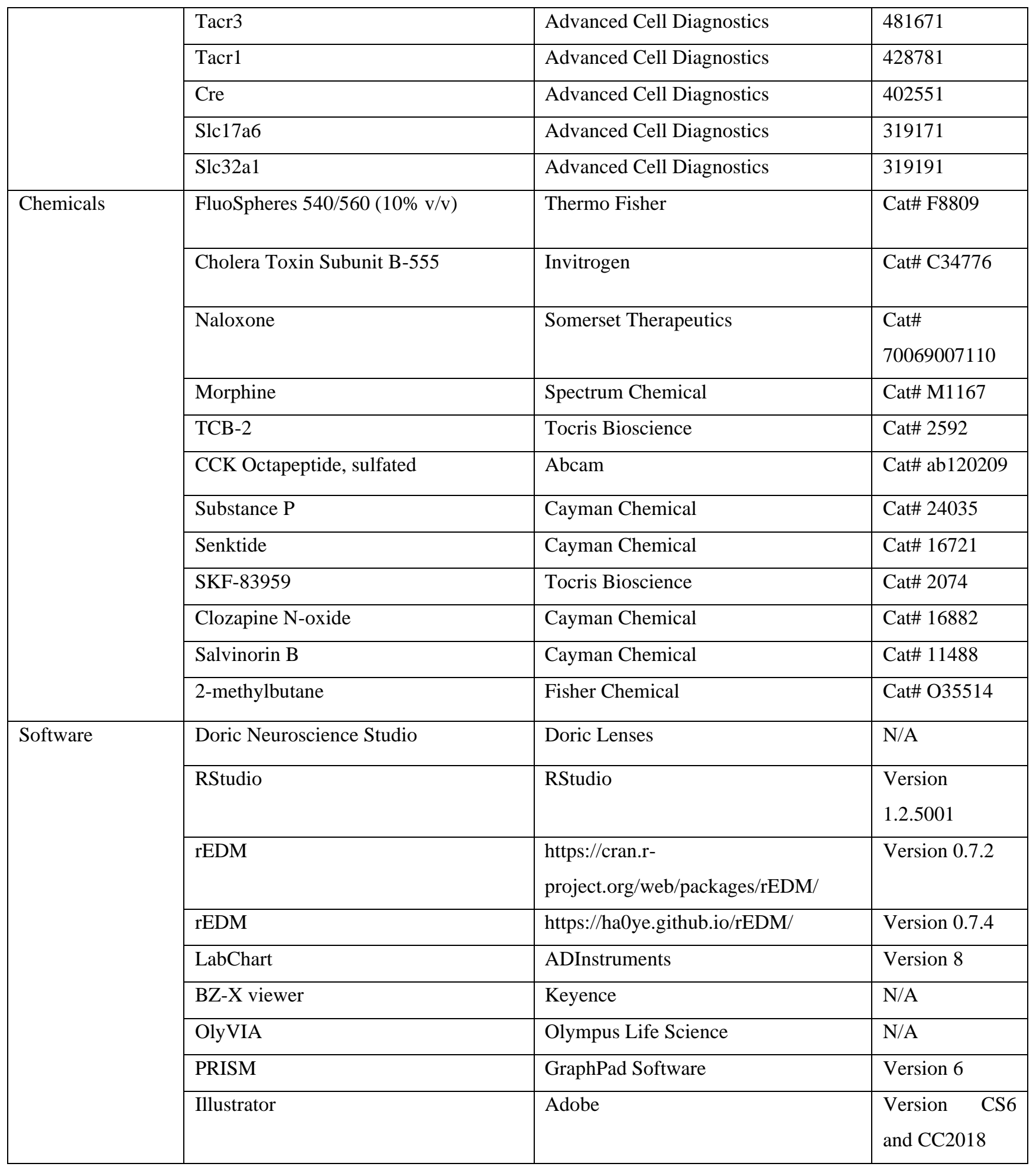

